800.20 (2) 8.

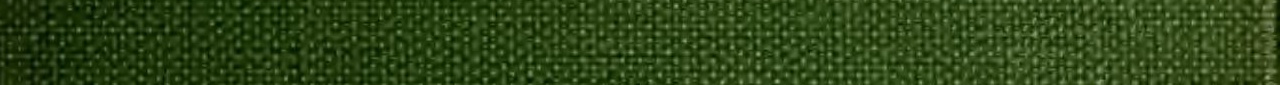
2030 


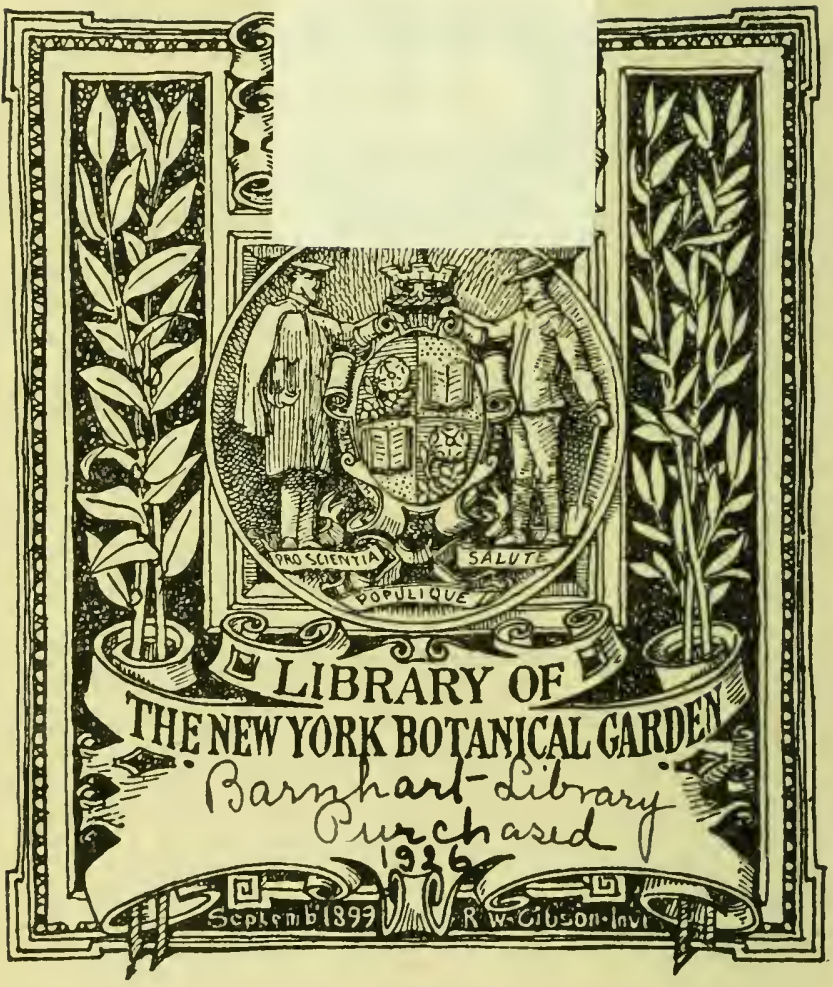




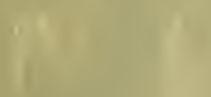

$$
\text { . }
$$



I Canifimo temonan

SYNOPSIS

\section{HYMENOPH YLLACEARUM.}



SY NOPSIS

\section{HYNENOPHYLLACEARUII.}

HOVOGRAPIILE IIJUS ORDINIS

PRODROMUS.

AUCTORE

R. B. VAN DEN BOSCH M. D.

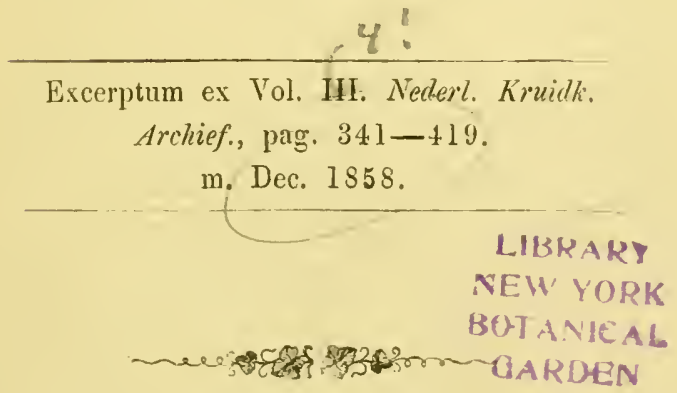

LUGi), BatavoruM APUD

JACOBLY HAZENBEKS CORN' FIIIUM.

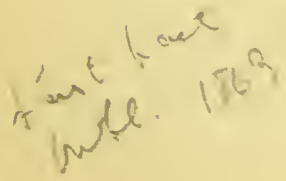




$$
\begin{aligned}
& \text { QL1 } \\
& \text { A1 } \\
& B 62 \\
& 1859
\end{aligned}
$$




\title{
SY NOPSIS
}

\section{Y II E Y O P II Y L L A CE ARU II.}

\author{
AUCTORE
}

R. B. VAN DEN BOSCH M. D.

Q!num anno 1855 Iymenophyllaceas Javanicas ut determinarem et describerem tradidisset am. ne Vriese, statim intellexi, me in hoc opere boni quid praestiturum non esse, nisi totius familiae notitiam simul mihi compararem. Mox autem studium illud adjumentum esse desiit. Imo, absoluta specierum Javanicarum Synopsi (Plantae Junghuhnianae I. p. $5 \%$ seq.), universalem illam cognitionem tam(juam novum stıdiorum scopum mihi proposui, cui adtingendo per ultimum biennium horas omnes impendi subsecivas, parcam medici praxin factitantis sor- 
tem. Meque non minus alliciebant plantulae, elegantia nescio an formarmm diversitate insigniores, quam incerta et confusa illas tractandi ratio, quae vel in optimorum PteridographoI'um libris offendebant. Patuit enim specierum distinctionem saepe fallacibus characteribus inniti, characteres contra optimos saepe negligi, studiumque hoc universe institui secundum methodum, vegetabilibus tam exiguis tamque tenerae structurae omnino non adaequatam.

Microscopio itaque usus, omnes species, quarum specimina habebam, accurate investigare statui idque inprimis egi, ut specierum ab auctoribus veteribus descriptarum notitiam mihi compararem. Non enim poteram quin mirarer, auctores recentiores hasce adeo neglexisse, ut, paucis exceptis, illis sint dubiae vel ignotae. Inter omnes autem constat, veterum testimonia in hac scientia non impune negligi neque decere excusationis causa afferre, illorum diagnoses nimia brevitate peccare dignoscendaeque speciei non semper sufficere. Propterea quantum potui eorum specimina aeque religiose ac recentiorum investigavi et characteribus, quos microscopii usus suppeditavit, novis magisque certis denuo in schedulis descripsi et in propriun usum delineavi.

Monographia Hymenophyllacearum hoc modo nata est, quam velim ut iconibus illustratam 
publico usui aliquando tradere possim. Interim ut aliquem studiorum fructum percipiam, inprimis vero ut anicis et fautoribus, qui varia auxilia obtulerunt, quomodo hisce sim usus, ostendam, Synopsin hanc in lucem edere constitui, quae, si quis Botanicorum eidem agro colendo praeter me operam dederit, quid egerim, quidve agendum reliquerim, ei declaret.

Quod ad Synopseos rationem attinet, patuca monenda habeo. Catalogi aridi taedio lectores ut parcerem, synonyma pauca specierumque patriam adjeci, quorum auxilio quid subintellexerim rei perito facilius patescat. Plurium specierum autopsiam consequi adhuc desidero. Nihilominus in Catalogum eas recipere non dubitavi quotiescumque ex descriptione vel icone de speciei auctoritate dubitare non licebat; quarum vero sive characteres sive affinitatem $e x$ iisdem eruere non potui, has tamquam ignotas suo quantumpote loco inserui.

Contextum frondis eo majoris habeo momenti, quo altius in hoc studio penetravi, characteresque propterea a cellularum forma, magnitudine, contentis mutuatos in descriptionibus semper inserui juxta illos, quos frondium forma, divisio, sororum dispositio el forma suppeditarunt. Cellulae autem, ut prius, dicuntur minimae, quibus lunen est usque $0,{ }^{\mathrm{mm}} 4$, dum idem parvis est $0,{ }^{\mathrm{mm}} 4-0,7$, mediacribus 
$0,{ }^{\mathrm{mm}} 7-0,8$, magnis $0,{ }^{\mathrm{mm}} 8-1,0$, maximis $u 1-$ tra $1,{ }^{\mathrm{mm}} 0$ : in cellulis elongatis diameter longitudinalis transrersali duplo est major. Quoad frondis divisionem monere oportet, frondem pinnalifidam dico, cujus rhachis utrinque ala foliacea lacinularum simili praedita est, pinnatam contra, quando illa sive est teres, sive laciniis decurrentibus tantum marginata; hoc in casu ala rhacheos sensim fit angustior pinnamque sequentem non attingit. Directio porro laciniarum determinatur terminis: erectus, patulus, divaricatus, horizontalis, deflexus secundum angulum plus minusve acutum, quem respectu rhacheos efformant. Stipitis frondisque dimensiones ad modum decimalem judicantur.

In distinguendis noris speciebus parcus fui. Imo plurimas in schedulis distinctas mihique minime dubias nunc reticere consultum videbatur. Magis enim, spccies ab aliis determinatas et descriptas microscopica methodo conprobatas tradere, cordi est. Si nibilominus justo nimium distinxisse alicui vidcar, ei in mentem revocare liceat praestantissimi sui temporis viri effatum, in hac familia praesertim neglectum: "pracstat enim, inquit, plantas cum non plena certitudine reri discriminis inter censum verarum stirpium conservare, quam inter varietates relegare disperituras" (IIAlder Ilist. stirp. Helvet. I p. 19\%.) 
Genera a Cl. Puest proposita, paucis exceptis, delenda, ino quae conserventur, emendanda esse, persuasum mihi habeo. Venarum decursus et distributio, in filicibus caeteris disponendis egregii, in Iymenophyllaceis vix alicujus est usus. Ilabitus contra est quaedan similitudo et ratio typica, quae mira constantia in magno saepe specierum numero rediens, genera in posterum statuenda divinare quasi sinit. At vero species antea melius sint cognitae, earum historia plenius sit illustrata, vitae ratio et distributio geographica diligentius sint observatae; nam, ut viri inter nostri aevi Botanicos praestantissimi El. Fules verba repetam: prana sunt nova genera, sine universali specierum cognitione et praecipue morphoseos historia."

Restat, ut gratum animum significem viris clarissimis, qui augendis et suppeditandis auxiliis studia mea aljuvarunt utque iis, quorum auxiliis, ut pensum hoc absolvere possim, opus mihi est, studia mea commendem. 


\section{H Y M E N O P H Y LLA CE A E.}

A. Trichomanoïdeae.

Cardiomanes Pr.

1. C. reniforme (Fonst.) Pr. Hym. p. 12; Trichomanes Fonst. Prodr. p. 84. Ilab. Nova-Zeelandia, Forst Frasen! Mosman! etc.

Feea Bory emend.

1. F. botryoüdes (Kuvlf.) Trichomanes Kavlf. Enum. p. 263 (1824); $F$. nama Bory Dict. d. sc. nat. VI. p. 447 c. icon. (1816).

Hab. Guyana Gallica, Poiteau!

2. F. spicala (Hedw.) in Wer. et Monr Beitr. (1805) I. p. 116 (nomen); F. polypodina Bory in Dict. d. sc. nat. VI. p. 
447 c. icon. (1846); Trichomanes spicisorum Desv. in Berl. Mag. V p. 329 et $F$. osmundoïdes DC. in Porr. Encycl. meth. VIII p. 65 - utrumque teste div. Kunze.

Hab. (Jamaica, St. Vincent), Trinidad! Guadeloupe! Guyana Gallica!

3. F. Boryi. Iymenoslachys diversifrons BOR in Dict. d. sc. nat. VIII p. 462 c. icon. Hab. St. Domingo! Guyana Gallica! (America centralis.)

Obs. Nomen triviale hujus et insequentis speciei, characterem quippe generis ideoque omnibus speciebus communem indicans, commutare ausus sum.

4. F. Humboldli. Trichomanes helerophyllum $\mathrm{H}$.

B. K. Nov. Gen. I p. 25.

Hab. Juxta ripas Rio negro, Hums;

Fl. Essequebo, Scromr. (n. v.)

5. F. Spruceana (Ноок.) Trichomanes Hook Cent. of ferns tab. 81 .

Hab. Rio negro, Spruce. (n. r.)

Neuromanes Trevis. ")

* Venae venulis spuriis transversalibus reticulatim conjunctae.

*) Nomen Preslianum (1843) retinere non licet propter homonymum genus Umbelliferarum a TorReY et GRAY in Flora of North-Amcrica anno 1838 propositum. 
1. N. Ileduigii. Trichomanes pinnaium HDw. gen. et sp. fil. fasc. I tab. IV fig. 1. ")

Ilab. Jamaica! St. Domingo, Sieb! Guadeloupe, L'Hermix! Trinidad Crüger! Guyana Gallica, Le Prieur! Surinam, Splitg! Kegel, Hostu! Brasilia, Mart! Clauss! Peruvia, Hhe! Venezuela, Fuxк et Sculim! (Rio Negro pr. S. Carlos, Нuив. en Bonpr..)

2. N. Kaulfussï. Trichomanes pennatum KaulF. Enum. p. $26 \%$.

Hab. (Jamaica, Trinidad. (Ноок.), Gu. yana Gallica, Снам.); Surinam, Splitg! Hotsu! Kegel; Guy, anglic., Schoub! Brasilia, Luscin., Makt! N. Grenada, (ocana), Scilum! (Mexico (S. Pedro Tepinapa) Liebu.), Guatemala, Keger!! (Peruvia (KzE).

Obs. Div. Kuxzen sententia de diversitate hujus et antecedentis speciei (Bot. Zeitg. 1817 p. 352) structura frondium egregie confirmatur. Perpetuae autem utriusque confusioni non nisi mutandis nominibus mederi posse, persuasum mihi habeo.

*) Tabulas in opere Hedwigii, ut citari possint, numeravi secundum ordinem quo editae fuerunt. 
3. N. Villaria (DC.) Pr. Hym. p. 19; Trichomanes DG. in Porr. Encl. meth. VIII p 65.

Hab. Surinam, Kappl Kegel! Spurg! Guyana gallica (t. Kze)

4. N. immersum 11. sp. Fronde pimata, pinnis e basi lata inaequali ovato-oblongis petiolatis, sessilibus vel sursum deorsumve rhachi latius breviusve adnatis ultima elongata oblongo-lineari inciso-lobato, margine irr'egulariter(saepe lacero-) dentato; venis crebris tenuibus s mplicibus furcatisve in marginem excurrentibus venaque marginali continua conjunctis, venulis transversalibus crebris; cellulis (forma et magnitudine ut in $N$. Kaulfussii) interaneis vero diffusis intermixtis globulis paucis; soris valde dispersis immersis, indusio parvo urceolato.

Rhizoma breve lıorizontale dense radiculosum, stipites fasciculatos debiles emittens; frons tenuis diaphana olivaceo-viridis.

A reliquis differt: Statura minore (stipes $0,06-0,1$ frons 0,07 longa, $0,02-0,0$ lata), consistentia frondis et structura, soris inmersis etc.

Hab. St. Domingo! de Tussic it IICr'J. FéE. 
* venulis transversalibus nullis.

5. N. abruptum Fée Mém. I p. 14 tab. I fig.

$5(18 \%) ; N$. IIostmannianum Kl. in

Linnaea XVIII p. 532 (1845).

Hab. Guyana Gallica Le Prièur!

(Surinam, Hosтu,; Brasilia? (t. Kze).

Cephalomanes $\mathrm{Pr} .{ }^{*}$ ) emend.

Rhizoma brevissimum, frondes fasciculati.

1. C. rhomboïdeum (J. Sm.) Trichomanes J. Sm. in Ноок. Journ. III p. A17; C. atrovirens Pr. Hymen. p. 18. tab. V. †)

Hab. Ins. Philippinat. Cum! Java, ZOLL!

2. C. Javanicum (Bl.) Trichomanes Bl. Enum.

II p. 22\%; C. oblongifolium Pr. Epimel. p. 19 tab. 10.

*) „Nomen genericum dignum alio, licet aptiore, permu„tare non licet." Linn. Phil. Bot. 243. Praecepto huicce persapienti, servando et hoc genere et Leptocionio, utrumque licet minime aptum sit, obte mperare decet.

+) Nomina plantis collectionum venalium antea non descriptis imposita, etiamsi nulla addita sit descriptio, iis quae alius quis iisdem plantis serius imposuit, prioritatis quam dicunt gratia, servanda esse persuasum mili habeo. Proptcre anominas Smithiana Hymenophyllacearum Cumingianarum anno 1841 edita Preslianis in monographia serius (scil. anno 1843) praeferenda esse censeo. Rem aliter sese habere simulac de nominibus manuscriptis collectionum privatarum publicarumve agitur, non est quorl moncam. 
Hab. Jara! Be., Zocr.; Ins. Philıpinae, Cus!

3. C. curvatum (J. Sm.) Trichomanes J. Sм. in Hook. Journ. III p. 417; T. asplenioïdes Pr. Hymen. p. 5 et 37.

Hab. Ins. Philippinae, Cumran!

4. C. Zollingeri v. D. B. in Pl. Jungh. I p. 552 (seorsim p. 8.)

Hab. Java! Soembawa! ZoLL.

5. C. Boryanum (KzE) Trichomanes Kze. Farrnkr. p. 237 tab. 97; T. alatum Bony in Dup. Voy. Bot. p. 282 tab. 38 fig. 2.

Hab. Ins. Carolinae, Mertens. (n. v.)

6. C. Singaporianum. Trichomanes Javanicum

Hк. el Grev. tab. 240 (non Bl.)

Hab. Singapore, Wallich (n. v.)

7. C. Madagascariense n. sp. Fronde oblongolanceolata pinnata, pinnis inferioribus horizontalibus contiguis, superioribus mediisque divaricatis imbricatis cunctis breviter petiolatis e basi lata cordata inaequali oblongis, margine inaequaliter dentato-serratis, apice rotundatis, venulis remotiusculis $1-2$ furcatis; cellulis maximis teneris elongatis hexaedris, parietibus rectis hyalinis, interaneis amorphis fuscescentibus seriatim radiatimque dispositis; soris subimmersis, mediotenus latius 
superne anguste marginatis, indusio cylindrico parum ventricoso, ore dilatato patulo recto.

Stipes triqueter glaber 0,1 et ultra longus, frons tenuis diaphana olivacea $0,15-0,18$ longa, $0,03-0,04$ lata, pinnae $0,010-0,016$ longae, $0,006-8$ latae.

Habitu et statura convenit cum C. Zollingeri $c$. et curvato, sororum forma cum $C$. rhomboïdeo.

Hab. Madagascar! Borrin in Herb. de Fraveueville.

** Rhizoma scandens, frondes sparsi.

8. C. dissectum (J. Sн.) Trichomanes J. Sн. in Ilook. Journ. Bot. III p. 417; T. dimidiatum Pr. Hym. p. 15 et 38 .

Hab. Ins. Philippinae! Cuming; Ins. Loo-choo (Oosima)! Wright.

9. C. Auriculatum (B⿺.) Trichomanes Bu. Enum.

II p. 225; T. Bélangeri Bony in Bêt.

Voy. Bot. p. 79 tal). 8 fig. 1.

Hab. Java! Béc.! Reinw.! Zor.l. Species ignota :

T. fuscum Bl. Enum. II, p. 225. Java. 
Trichomanes S.r.

"Rhachis s. costa nulla, venae furcato-ramosae.

a. Stipes simplex.

1. T. parvulum Porr. Encl. meth. VIII p. 4.4;

T. Sibthorpiö̈des Bony in Wiltid. sp.

pl V p. 498 (testibus Hook, et Kze.

Hab. Nadagascar, Thovans; Ins. Mascarenae, Bonr (n. v.)

2. T. saxifragö̈des Pr. Hym. p. 16 et $39 ; T$.

parvulum BI. Enum. p. 223 (excl. syn.)

Hab. Ins. Philippinae! Cumsa; Java!

ZolL.; Rawak! Gaudich.

3. T. Thouarsianam Pr. Hynı, p. 16 et 10.

Hab. Ins. Borboniae, Tuouars. (n. v.)

4. T. digitalum Sw. Syn. p. 370 et 1.22; T. lanceum Willd. Sp. pl. V p. .500.

Hab. Ins. Mascarenae! Sieder, Bony, etc.

5. T. flabellatum. T. digilatum BL. (non Sw.)

Enum. p. 22! (excl. Syn.)

Hab. Java! Bu., ZoLl.

6. T. palmalifidum C. Metr. in Bot. Z1g. 185\%

p. 732; T. digilatum KzE BoT. Zig.

1847 p. 302 (non Sw. nec. Bu.)

Hab. Java! Zoll., v. Gèsk.

7. T. nitiduhum v. B. B. in Pl. Jungh. I p. 5 1.7 (seorsim p. 3.)

IJab. Java! v. Gesk, JengrI. 
8. T. dicholomum Kze in Bot. Ztg. 1848 p. 285. Hab. Java! Zoll.; 'Taïti! Vieill et Plancir.

$\beta$. stipes prolifero-ramosus.

9 T. proliferum BL. Enum. p. 224; T. palmalum. Pr. Hym. p. 16 et 39.

Llab Java! BL., Zoll. Teissm.; Ins. Philippinae! Cuming.

10. T. minutum BL. Enum. p. 223 ; T. parvulum Kze in Zolt.! Verz. p. 45. Species ignota.

T. bifolium BL. Enum. II 22\%. p. Java.

** Rhachis venas flabellatim vel pinnatim emittens.

a. Sori primitus terminales.

$\alpha$. Venulae spuriae,

† secus marginem junctae.

11. T. cuspidatum Wruld. Sp. pl. V p. 499;

T. Bojeri Hook. et Grev. tab. 155. Hab. Ins Mascarenae! Thouars, BélanGEr, Bory, Borvin, alii.

12. T. Ilookeri Pr. Hym. p. 16; T. muscoüdes Ноoк. et Grev. Lab. 179 (non Sw.) Hab. (Janaica), Cuba! Gnyana Gallica! (Peruvia?) 
13. T. erosum P. B. Fi. d'Oware et de Benin II p. 79 tab. 109 fig. 3.

Hab. Africa occidentalis, P. B. (n. v.) 14. T. membranaceum L. Sp. pl. Ed. $2^{\mathrm{a}}$ p. 1560. Hab. Antillae! Guyana Gallica! Columbia!

\section{tt liberae.}

15. T. Petersii A. Gray in Sillin. Am. Jomrn. sc. and arts $2^{\mathrm{e}}$ Ser. 1853. p. 326 (sec. Ноок. Cent of ferns tab. 86.)

Hab. Amer. Sept!,(Alabama) Peters. 16. T. sublimbatum C. MüLt. in Bot. Ztg. 185\% p. 737; T, Hookeri Kze in Bot. Ztg. 1848 p. 285 (non Pr.); T. Muscoïdes id. in Bot. Ztg. 1846 p. 476 non Sw.)

Hab. Java! Zippelius, Zollixger. Species ignota:

T. adiantinum Bory in Bél. Voy. Bot. p. 78.

ß. Venulae spuriae nullae.

S 1. Pseudachomanes Pr. (T. simuosum etc.)

† Frons pinnatifida.

17. T. simıosum Rich. in Willd. sp. pl. V p. $502 ; T$. Quercifolium Desv. in Berl. Mag. V p. 328 (non Hook. et Grev.); T. Poeppigii Pr. Hym, p. 16 et $\mathbf{4 1}$ (teste ipso ) 
IIab. Guadeloupe! Trinidad! N. Grenada! Venezuela! (Peru), Mexico!

18. T. incisum KLf. Enum. p. 261; T. cognatum Pr. Hris. p. 16 et $\$ 1$ (teste Kze.) Hab. Brasilia! Cınısso, Bory, Mart, Berrich, etc.

19. T. holopterum Kze Farrnkr. p. 185 tab. 7 fig. 2.

Hab. Guadeloupe! Martinique! S'. Vincent! Guyana Gallica!

20. T. Bancrofti Ноok. et Grev. tab. 20'; $T$. coriaceum Kze Linn. IX p. 105 et Analect. p. 46 tab. 29 fig. 1.

Hab. Surinam! Guyana Gallica! et anglica! Brasilia! (Jamaica, S`. Vincent.)

Species ignola :

T. arbuscula Desv. in Ann. Soc. Linn. Paris VI p. 326, Guyana.

$\div$ frons bipinnatifida.

21. T. adsrendens Kze Farrnkr. p. 186; Bot. Ztg. 1817 p. 320.

Ilab. Ins. Martinique! MIle . Rivoire.

22. T. alratum Sw. Fl. Ind. occid. III p. 1732 [non Hnok.]; Prum. Folig. p. 73 tab. $50 \mathrm{D}$.

Jab. ( $\mathbf{S}^{\mathrm{t}}$. Domingo), Jamaica! 
23. T. ptilodes. T. alatum Hook, et Grev, tab. 11 (non Siv.)

Differt a praecedente: fronde duplo altiore et latiore apice lanceolata, la ciniis latioribus magis invicem remotis, lacinulis superioribus linearibus, venulis remotioribus, cellularum magnitudine et forma, indusiis magis elongatis cylindricis orificio bicorni, etc.

Hab. Jamaica! Guadeloupe! Venezuela!

21. T. attenuatum Ноок. sp. fil. I p. 122 tab. $39 \mathrm{C}$.

Hab. St. Vincent, Jamaica, Dominica. (n. v.)

Obs. Specimina non vidi; genuinum vero $T$. alatum Sw. sistere, ex habitu frondis sororumque forma etc. verosimile videtur.

tit frons tripinnatifida.

25. T. Lindeni Pr. Epimel. p. 11 tab. 6 (1849); T. macroclados Kze Farnnkr. II p. 72 tab. 130 (1851); T. scandens Ноок. sp. fil. I p. 140 (nec L. nec Sw.)

Hab. Jamaica! Linden; Cuba! LiNDen, Wright! Polto-ricco! (Mexico?) Obs. Frons rectius pinnata dicitur 
prinnis bipinnatifidis; rhachis enim apice excepto nuda est et teres.

26. T. Bicorne Ноок. Cent. of ferns tab. 82. Obs. Affinitas speciei non visae mihi latet.

IIab. Rio negro, R. Spruce. (n. v.)

5. 2. Achomanes Pr. (T. crispum L. etc.)

S) frons pinnatifida. "]

27. T. $\operatorname{crispum}\left(\right.$ L. Sp. pl. Ed. $2^{\mathrm{a}}$ p. 1560 (non Hedw. nec Hook); Plom. Foug. p. 67 tab. 86 .

Hab. (Martinique; Plum.) Portoricco! Scuwanecke.

28 T. fastigialum Sieb. FL. Martin. N.?;

Syn. fil. N. 144; T. crispum Hedw.

Gen. fil. tab. IV fig. 2 (non L.)

Hab. Jamaica! Sw.; Martinique! Sieb.; St. Vincent!

29. T. accedens Pr. Epimel. p. 14; T. crispum Hook et Grev. tab. 12. (non L.) Hab. Guadeloupe! L’Herminier;

*) Speeies plures hujus sectionis, dificiente speciminum bonorum copia, mihi non satis notae sunt. Rhizoma breve adscendens frondesque fasciculati in nonnullis, in aliis rhizuma horizontale frondesque sparsi egregium subdivisionis characterem suppetere videntur. 
30. T. pellucens Kze in Linn. IX p. 104; Farnkir. p. 158 tab. 68 .

Hab. Brasilia! v. Mart., M'Rae; Peruvia! Poeppig, Lechler; (Guyana Allglica, Бсномв.)

31. T. cristalum Kaulf. Enum. p. 265.

Hab. Brasilia! Ganos.; Surinam! Weigelt, Kapler, Guyana Anglica!

Schomb.

32. T. pilosum Raddi Fil. Brasil. p. 63 tab. $九 9$ fig. 1; T. laxum Kl. in Linn. XVIII p. 530 (teste Kze.)

Hab. Brasilia! Radd, uE Mart., Princ. Neovid, alii; Columbia! MontTz; Guyana Gallica! Le Prieur.

33. T. Martiusii Pr. Hymen. p. 15 et $36 ; T$. pilosum Mart. Icon. sel. p. 105 (excl. Syn.) tab. 68 (fig. dextra).

Hab. Brasilia, de Mart., Guyana Angl., Sсномв.? Surinam, Hostм.! Kеgel, Splitg! (vix. r.)

34. T. Plumula Pr. Hymen. p 15 et $36 ; T$. pilosum Mart. I. l. (fig. sinistra).

Hab. Brasilia, de Makt! Guyana Angl., Sсномв!

35. T. plumosum Kze in Linn. IX p. 104.

Hab. Peruvia! Poeppig.

36. T. Haenkeanum Pr. Hymen. p. 15, 36 et 65; T. crispum $\mathbf{P}_{\mathbf{R}}$, Reliq. Haenk. I 
p. 69 (excl. syn. teste ipso.)

Hab. Peruvia! Dombey, Haenke; Bolivia! Weddell.

37. T. Sellowianum PR. Hymen. p. 15 et $3 \bar{\nearrow}$, Hab. (Brasilia, Sllow, Gardxer.

PoHL; Surinam, Kegel); Columbia? Funck et Schrim..

38. T. eriophorum (PR.) Ragatelus Pr. Epimel. p. 18 tab. 9.

Hab. Guyana Anglica, Sсномв. (n. v.)

t† frons bipinnatifida v. pinnato-pinnatifida.

39. T. Kaulfussii Нook. et Grev. Icon. App. p. b; T. lucens ibid tab. 10 (non Sw.) Hab. Ins. Antillarum (Jamaica, $S^{t}$. Vincent, Trinidad! Dominica, $\mathbf{S}$. Kitts, $\mathrm{S}^{\imath}$. Thomas, Martinique! Porto-ricco!

Guadeloupe!) (Guyana, Parker.)

40. T. lucens Sw. Syn. p. 143 (nоn Ноок.); Hevw. Gen. fil. tab. V.

Hab. Jamaica! Sw., Antilles (s. loco spec.) de Tussac!

41. T. splendidum. T. lucens Ноок. Sp. fil. I p 138 tab. 41 A. (excl. Syn.)

Hab. Sa. Fé de Bogota! Hartw.; Peruvia! Leciller.

42. T. Lambertiamum Ноок. sp. fil. I p. 139 tab. $41 \mathrm{~B}$.

Hab. Peruvia, Ruz et Pavox. (n. v.) 
43. T. crinilum Sw. Fl. Ind. occid. III p. 1730;

Rayatelus Pr. Hymen. p. 16.

Hab. Jamaica! Sw., Mevz., Purdue;

(St. Vincent, Gullo.); S'. Domingo!

de Tussac; Guadeloupe? lHeryin.

Species ignotae:

T. pallidum Be. Ènum. II p. 225. Java.

T. elatum Dewr. in Ann. Soc. Linn. Paris. VI

p. 327. America tropica.

b. Soris primitus axillares lateralesve.

a. Craspedoneuron (T. album Bl. elc.)

44. T. album Bl. Enum. II p. 226.

Hab. Java! Reisw., Bl., v. Gesk.;

Ins. Philippinae! Cur.

45. T. Braunii v. D. B. in Pl. Jungh. I p. 550 (seorsim. p. 6); T. album Kze Bot. Ztg. 1846 p. 476 (non $B L$.)

Hab. Java! Bu.? in Herb. AL. Br.

46. T. glaucescens v. v. B. in Pl. Jungh. I p. 551

(seorsim p. 7); T. album Kze Bot. Ztg.

1817 p. 350. (non Bu.)

Hab. Java! v. Gesk., Zoll.

47. T. glaucofuscum Hook. sp. fil. I p. 128 ; tab. 40 A; T. aculum Pr. Hriev. p. 16 et 12. IIab. (Ins. Australes), Philippinae!

Ceylon! (Penang.) 
B. Crepidomanes. (T. humile Fonst. etc.)

48. T. humile Forst. Prodr. N. 464; T. Luzonicum Pr. Hymen. p. 16 et 42.

Hab. Ins. Societatis! Australes! Philippinae!(Otaheite), Taït! Java!

49. T. Endlicherianum Pr. Epimel. p. 10 tab. $5 \mathrm{~A} ; \mathrm{T}$. humile $\beta$. Ноок. sp. fil. I p. 123.

Hab. (Ins. Norfolk); Nov. Zeelandia! 50. T. intramarginale Ноoк. et Grev. tab. 211. Hab. (Ceylon, Lindere); Hong Kong! Wirigit.

51. T. Colensoi Ноок. Cent. of ferns tab. 79. Hab. Nov. Zeelandia, Colenso (n. v.) Vix hujus loci!

52. T. Schmidianum Zenk. in Taschn. Diss. p. 3' tab. I fig. 1.

Hab. Malabaria (Oottacamund), Scrimid!

53. T. depauperalum Bory in Duperr. Voy. Botan. p. 283.

Hab. Ins Carolinae! Duperr.

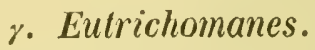

$+T$. pyxidiferum L. etc.

5'. T. pyxidiferum L. sp. pl. Ed. $2^{\text {a }}$ p. 1561 (excl. Syn.); Plum. Forg. p. 74 tab. 50 E. (non aliorum.) 
Hab. (St. Domingo, Plun.) Guyana Gallica! Lepreueu.

55. T. Brasiliense Dêsv. Mém. Soc. Linn. Par. VI p. 328 tab. VII fig. 4; T. pyxidiferum Hedw. gen. fil. Lab.III. fig. 2. Hah. Brasilia! v. Mart., Wedoell; Venezuela! Funck et Scilum.

56. $T$. emarginalum Pr. Epimel. p. 11 tab.

B; T. pyxidiferum $\beta$. Ноок. sp. fil. i

p. 124 (р. p.)

Hab. Brasilia! De Mart., Beyrucu.

57. T. leptophyllur. T. pyxidiferu:n Ноoк. et Grev. tab. 206; Hook. sp. fil. I [). 121 (fide specim.)

Ilab. St. Vincent! Martinique! Guadeloupe! Jamaica! St. Domingo! Cuba! 58. $\boldsymbol{T}$. oluaceum KzE teste Kr. in Linn. XX p. 437. (a neutro descriptum.)

Hab. Columbia! Montrz; Nov. Grenada! Schlı; Mexico (Huatusco)! ScIIAFFner.

59. T. cavifolium C. MüLl. in Bot. Ztg. 185 ' p. 753 .

Hab. Mexico, Leiboed! Schlede! SchaFFNeK!

60. T. Lechleri. T. pyxiliferum? Kze in Lins. IX p. 106.

IIab. Peru! Lechl.; Quito! Jniesox. 
61. T. exsectum KzE Anal. p. 47 tab. 29 fig. 2. Hab. Ins. Juan Fernandez! C. GaIJ; (Ins. Chiloë, Cunung; Valdivia, BridGES.)

62. T. angustatum Carm. in Linn. Trans. XII p. 513.

Hab. Ins. Tristan d'Acunha, Carm.(n. v.)

63. T. tenerum Spr. Syst. veg. IV p. 129.

Mab. Brasilia, Brancuet! de Mart.! Regnell ! Beyrich! etc. Peru, Matth! Quito, Jameson! Saettone!

Obs. T. angustato non viso, $T$. tenerum distinctum ab illo habere debeo, dum utriusque specimina comparare licuerit. Specimina Brasiliensia, praesertim quae a Martio et Beyricuro lecta vidi in Herb. am. Sonder el frondis divisione et indusiorum forma cum icone $\boldsymbol{T}$. angustali satis bene convenire, non negandum; quae vero a Regneluro lecta ibidem vidi, qwaeque omnino sistunt $\boldsymbol{T}$. fulvum Kl. (fide Herb. Mus. Par.) characteribus haud levibus recedunt.

61. T. Schiedeanum C. MüLl. in Bot. Zlg. 1854. P. 716.

Ilab Mexico, Scuede! Scunffaen! etc.

6.). T. runciforme Scuis. Farmkir. p. 135 tab. 
134 (nomine T. pyxidiferi per errorem inscripta).

Hab. St. Domingo, (n. v.)

66. T. trichö̈deum Sw. Syn. p 1.14; T tenellum Hedw. fil. gen. tab. III fig. 1; T. angustissimum Pr. Epimel. p. 18 tab. $8 \mathrm{~A}$.

Hab. Jamaica! Sw; Columbia (Venezuela! Montz, Frusck et Scilim, Nov. Grenada! Sciulu.)

67. T. cellulosum Kr. in Linn. XVIII. p. 531. Hab. Guyana Anglica, Schour. (n. v.) 68. T. melamotrichum Schltde. in Linn. X p. 553. Hab. Prom. bon. spei! ZeYHer, Drège, etc.; Ins. Borboniae! Borr, Boivin, Ricisard.

$\uparrow+T$. radicans Sw. etc.

69. $T$. radicans Sw. Fl. Ind. occid. III p. 1736 ; T. scandens Hedw. Gen. fil. tab. VI. (non L.)

Hab. Jamaica! specim. Acusr. in Ilerb. Sonder.); Cuba? Wright! Mexico? ScIlafFN.

70. T. exmium KzE in Bot. Ztg. 1846 p. 17S; 18.4 p. 350 (nomen); T. ambigum Kl. in Linn. XVIll p. 531 (nomen). Hab. Columbia (Venezuela! Monitz; N. Grenada! Sculmi). 
Obs. T. ambigunum Sieb. (Syn. fil. N. 143) a Prescro sancitum, dubia mihi species quandoquidem specimina modo viderim juvenilia et sterilia; ex habitu vero et contextu $T$. eximio ?affinius crediderim quam $T$. radicanti, cujus synonymon habent auctores.

71. T. Luschnatianum Pr. Hymen. p. 16 et 45.

Hab. Brasilia, (Bahia! Luschnat, Sierra de Registro! Mar't. Bens.) alii.

obs. Specimen quod vidi authenticum in Herb. Cl. Movgeot in diagnosin omnino non quadrat; convenit autem illud cum Mart. Herb. Fl. Brasil. N. 389. Differunt haec a $T$. radicante praeter notas externas, cellulis majoribus opacis firmis elongatis irregularibus (saepe quasi tetraëdris) interaneis obscure viridibus, cellulis marginalibus duplo minoribus (lacinulas lentis ope visas marginatas efficientibus).

72. T. scandens L. Sp. pl. Ed. 2a. p. 1562 ; Sw. Fl. Ind. or. III p. 1737; T. Kunzеапит Ноок. sp. fil. I p. 127 tab. $39 \mathrm{D}$.

Hab. Columbia Linoen! Moritz! Funck et Scillu! (Peru? Poepr., Matrin.) 73. T. Morilzï n. sp. Fronde sessili lanceolata 
bipinnatifida, laciniis primariis patulis divaricatisve remotis (imis exceptis) lere acquidistantibus e basi inaequaliter cuneato-lanceolatis apice productis pinnatifidis, secundariis strictusculis remolis 2-3 furcatis, lacinulis fastigiatis abbreviatis apice cmarginato-bifidis rotundatis; cellulis mediocribus vel parvis firmis opacis hexaedris vix elongatis interaneis obscure viridibus amorphis marginem versus obscure granulosis, soris e cylindrico ventricosis infundibuliformibus ore integro reclo parum dilatalo. Frons $0,5-0,35$ longa, $0,06-0,07$ lata firma rigida opaca obscure viridis.

Hab. Columbia, (Col. Tovar! MoRitz; N. Grenada 9000'! Scinıм.)

74. T. abrotanifolium n. sp. Fronde sessili vel subsessili lanceolata tripinnatifida, laciniis primariis patulis inferme distantilus sursum sensim approximatis superne contiguis e basi cuneata lanceolatis bipinnatifidis, secundariis stricliusculis cuneato-oblongis lanceolatisve inferioribus pinnatifidis superioribus dichotomis, lacinulis cunctis angustis strictis elongatis; cellulis magnis elonyatis hexaëdris teneris intcraneis lacte 
viridibus amorphis, marginem versus abbreviatis minoribus distincte granulosis; soris cylindricis elongatis superne in orificium undulato-repandum dilatatis. Frons 0,4 et ultra longa, 0,080,10 lata rigida virgata opaca obscure viridis.

Hab. Brasilia! De Mart. in Herb. Sond.

75. T. colluriatum n. sp. Fronde breviter stipitata lanceolato-lineari bipinnatifida, laciniis primariis divaricatis horizontalibusque remotis imis distantibus oblongis pinnatifidis, secundariis patulis divaricatisve contiguis e basi cuneata oblongis antice latioribus truncatis inciso-dentatis; cellulis parvis vel mediocribus firmis opacis hexaëdris non elongatis interaneis obscure viridibus granulosis; soris e cylindrico clavatis ore subilo in limbum amplum undulalum dilatato. Frons usque 0,3 longa, 0,05 lata rigidula opaca obscure viridis.

Hab. Mexico! (Tabasco) 300') Linden. 76. T. speciosum Wiı.Ld. Sp. pl. V.p. 514; T. radicans Ноок. Sp. fil. I p. 125 p. p. (non Sw.); T. speciosum var. Andrewsii Newm. Ilist. p. 315. 
Hab. Ins Canarienses! Madeira! (Ins. Azoricae), Ilibernia!

77. T. brevisetum R. BR. Hort. Kew. V p. 529; Iymenophyllum alatum Engl. Bot. tab. 1417; T. speciosum Newm. Hist. p. 305 .

Hab. Madeira! Hibernia!

Obs. Contextu frondis certo a $T$ specioso diversum est. Specimina vero, quibus sum usus, quippe diverso evolutionis stadio collecta, specierum diversitatem non extra omne dubium ponunt. Res denuo notaeque melioris auxiliis indaganda.

78. T. striatum Dos Prodr. Fl. Nepal. p. 1.

Hab. Nepal! (specimen auctoris ex dono ill. Lambert in Herb. Sonder).

79. T. Davalloödes Gaudich. in Voy. Uranie Bot. Cryptog. p. 378.

Hab. Ins. Sandwich(Woahoo)! M'RAE in $\mathrm{H}$ rrb. Sond. (Comm. Cl. Lindiey). Species ignotae:

T. umbrosum Wall. Cat. N. 165. Nepal.

T. venustum Desv. in Ann. Soc. Linn. Paris. VI p. 328. Brasilia.

T. diaphanum H. B. K. Nov. gen. p. 25. Venezuela.

T. anceps Wall. Cat. N. 166. Nepal. 
80. T rupestre Raddi (Hymenophyllum) Fil. Brasil. p. 67 tab. 80 .

Hab. Brasilia, Raddi, de Mart! Pohl! Weddela! alii.

Obs. Species insignis! sterilis modo hucdum lecta, at e contextu frondis vix dubie inter Trichomanes enumeranda.

81. T. Ankersii Park. in Нook, et Grev, tab. 201.

Hab. Guyana (Batava! Host.r., Anglica, Park., Schoum.; Gallica! Le Prieur); Trinidad! (specim. Ноок.) in Herb. de VR.)

82. $T$. brachypus Kze in Linn. IX p. $105 ; T$. radicans Hook. et Grev. tab. 218 ; T. subsessile Splitg. in Tijdschr. v. nat. gesch. VII p. 437.

Hab. Peru! Pöpp.; Brasilia, BranChet! de Mart! Guyana (Batava, Splitg! Kappl.! Gallica, Le Prieur, Anglica, Sсномв.); (Trinidad, LocкII., $\mathrm{S}^{\mathrm{t}}$. Vincent, Guild.)

Obs. Unam alteramve speciem hic esse confusam suspicari quidem, at deficiente speciminum copia affirmare non licet. 
H†t T. rigidum Sw. etc.

Americana.

83. T. rigidum Sw. Fl. Ind. occ. III p. 1738; HEDw. gen. fil. tab. II.

Hab. Ins. Antillarum (Jamaica, Domingo! Portoricco! Guadeloupe! etc.) Columbia (Venezuela! N. Grenada!) 84. T. Mandioccanum Raddi Fil. Brasil. p. 6' tab. 79 fig. 2 ; ? T. firmulum. Pr. Hymen. p. 16 et 46.

Hab. Brasilia, Radni, Mart ! ClauSex! Weddell! alii.

Obs. Cl. Prest, speciem suam rhachide et stipite nudis (non marginatis) a $T$. rigido differre perhibens, eo ipso hoc sibi ignotum esse, testatur.

85. T. compressum Lesv. in Berl. Mag. 1811 p. 329 .

Hab. Domingo! Poiteau.

86. T. Prieurii Kze Anal. p. 48; T. anceps Ноок. sp. fil. I p. 135 (p. p. et excl. $\beta$. Hab. Guyana Gallica, Ricr., LE Prieur (Anglica, Schoub.); Brasilia, dé Mart! Weddell; Peru! Lechl.

87. T. anceps Hook. sp. fil. I p. 135 (excl. $\beta$ ) tab. 40 C. 1.

Hab. Jamaica! Guadeloupe! Trinidad! 
Obs. Plures indubie hic latent species, non vero nisi examinatis speciminibus completis e variis regionibus probe dignoscendae. Equidem licet viros Cl. Ноoker et Kunze, quisque nomine a se proposito, idem subintellexisse persuasum mihi habeam, ad designandum speciminum, quae vidi, typum duplicem nominibus illorum mutato sensu uti, quam novum proponere, malui.

88. T. Daucoïdes Pr. Epimel. p. 12 tab. 7. Hab. (America centralis, Fridricustilal); Martinique! Bêranger.

89. T. lenuifolium Gav. (Prael. n. 697); Sw. Syn. p. 144.

Hab. Ins. Chiloë! NÉE. Species ignota:

T. mtermediùm Kaulf. Pr. Hymen. p. 16. Patria ?

* Capensia et Mascarena.

90. T. Drègei; T. rigzdum Kze in $\mathrm{Pl}$. acotyl. Afr. austr. p. 73 (excl. omn. Syn.)

Hab. Cap. bon. spei! Drége; Port Natal! Gueinblus.

91. T. Achilleaefolium WiLld. sp. pl. V p. 512;? T. slylosum Porr. Encycl. Bot. VIII p. 32. 
IIab. Ins. Mascarenae! Siek,, Borr, Borvis, alii ; Madagascar! 'Thouars.

92. T foeniculaceum Bony in WiLto. sp. pl. V p. 511;? T. parviflorum Porr. Encycl. méth Vill p. 83.

Hab. Ins. Mascarenae! Commers., Bonr, Borvin, alii; Madagascar! Thouars. 93. T. giganteum Bory in Willd. sp. pl. V p. 514 .

Hab. Ins. Borboniae, Bony!, BéL! Borvin! alii.

94. T. Lamarisciforme Jace. Coll. III p. 285 tab. 21 fig. 2; T. tamariscinum Boliy in BÉL. Voy. Bot. p. 81 ; ? T. parviflorum PoIr. Encycl. meth. VIII p. 83. Hab. Ins. Borboniae! Coumers., BéL., Borvin; Madagascar! Thouars.

95. T. meifolium Bony in WinLd. sp. pl. V p. 508.

Hab. Ins. Borboniae! Borv., BÉL., de Hell, alii.

96. T. lanceolatum Polr. Encycl. meth. VIII p. 83. Hab. Madagascar! Thounss (in Herb. Mus. Par.)

97. T. longisetum Bory in Willd. sp. pl. V p. 510 .

Hab. Ins. Borboniae! Bory, De MontBuison, alii; Java! Zoll.

98. T. Borimi n. sp. Fronde ovato- vel oblongo- 
lanceolata bipinnatifida, laciniis primariis e basi latiore lanceolatis contiguis vel remotiusculis, superioribus patulis, mediis inferioribusque divaricatis imo deflexis pinnatifidis, secundariis e basi angustata oblongis contiguis patulis vel erectiusculis irregulariter incisis (vix lobatis) inaequaliter obtuse dentatis, venis pinnatim ramosis, venulis ultimis furcatis parallelis densissimis, cellulis (aegre distinguendis) opacis mediocribus imo magnis valde inaequalibus irregularibusque elongatis, parietibus flexuosis incrassatis, interaneis amorphis diffusis spissis olivaceis, soris in laciniarum secundariarum incisuris immersis lateralibus, indusio cylindrico parum ventricoso incurvo orificio parum ampliato undulato, receptaculo setaceo indusio duplo et quod excedit longiore. Rhizoma brevissimum adscendens, radiculis ramosis, pariter ac stipes compressus apice pinnis infimis decurrentibus anguste marginatus rhachisque et venae, paleaceo-hirsutum, stipes 7 centim. longus, frons $10-14$ centim. longa, 5-7 centim. lata opaca rigida olivaceo-fusca. 
Affinitate proximum T. elongatum, quod differt: glabritie, stipite rhachibusque teretibus, structura frondis et colore, directione pinnarum, pinnulis lanceolatis profunde lobato-incisis etc.

Hab. Ins. S ${ }^{\text {te }}$ Marie (Madagascar) Boivin in Herb. ne Franguevilde.

Species ignotae:

T. myriophylhum Desv. in Ann. Soc. Linn. Paris. VI p. 329. Madagascar.

T. Thuyoides Desv. ibid. p. 328. Bourbon.

T. cypressoïdes Desv. ibid. p. 330. Ins. Sechelles.

T. Alchemillaefolium WaLL. Cat. N. 159. Mauritius. ${ }_{*}^{*}$ Indica et Malasica.

99. T. obscurum Bc. Enum. II p. 227; T. millefolium Desv. Mém. Soc. Linn. Par. VI p. 329; Didymoglossum longisetum Pr. Hym. p. 23 et 49.

Hab. Ceylon! (sp. Ноок. in Herb. de Vr.); Java! Reinw., v. Gesk., Zolu.; Ins. Philippinae! Commenson, Comra; (Sincapore, Lobr, Waldicir); M. Nilagirici! Pernottet; Ins. S ${ }^{\text {le }}$ Maric de Madagascar! Boıvin.

100. T. papillatum G. MüLL. in Bot. Ztg. 185 \% p. 751. 
Hab. Ins. Philippinae! Cuming.

101. T. maximum BL. Enum. II p. 228 (excl. var. $\beta$.)

Hab. Java! Bu., ZoLc.; Ins. Philippinae! Cuming.

102. T. Millefolium Pr. Hymen. p. 16 et 43 ; T. anceps $\beta$. Ноок. sp. fil. I p. 135 tab. 40 C. fig. 3.

Hab. Ins. Philippinae! Cuving; Java! Zolv.

103. T. Apüfolium Pr. Hymen. p. 16 et $44 ; T$. myrioplasium Kze in B. Z. 1846 p. 477. Hab. Ins. Philippinae! Cumrng; Java! v. Gesk., Zoll., Jungir.

10\%. T. gemmatum J. Sir. in Ноок. Journ. III p. 416 .

Hab. Malacca! Cuming.

105. T. eminens Pr. Hymen. p. 16 et 44.

Hab. Ins. Philippinae! Cumsrg.

106. T. Pluma Hook. cent. of ferns tab. 97.

Hab. Borneo, Loвb. (n. v.) Differtne revera a $T$. longiseto Вону?

Species ignota :

T. bifidum Vent. in Willd. Sp. pl. V p. 511. India orientalis.

*** Australica.

107. T. Baueriamum Ende. Prodr. Fl. Norf. p. 17; T. meifolium $\beta$. Ноок. sp. fil. Ip. 137. 
Hab. Ins. Norfolk, B.uver; Ins. australes, Benn., NıGut.; India, Ward. ; teste Hooker (n. v.)

108. T. strichum Menz. Ноок. et Grev. tab. 122.

Hab. Nov. Zeelandia, Mexz., Cuxv. (n. v.)

109. T. elongatum Curv. Ноoк. sp. fil. I p. 13 \% Hab. Nov. Zeelandia, J. D. Hook! Sıxcl! Mlossu.! F. MüLl.! alii.

110. T. polyanthos Hook. Ic. pl. tab. 703; sp. fil. I p. 138.

Hab. (Ins. australes, Nicnt.); TAïti! Vieill. et Plaxch. (in Herb. Moug.)

\section{Desciscentes :}

111. T. Smitlii IIоoк. Icon. pl. tab. 704 ; Abrodiclyon Cumingii Pr. Ilymen. p. 20! tab. 7 (??)

Hab. Ins. Philippinae! Cumisg.

112. T. venosum R. Br. Prodr. fl. nov. Holl. p. 915 ; Phlebodium Brownii v. D. B. in Sched.

Hab. Nova Hollandia! Nova Zcelandia!

113. T. cuespitosum (Gaud.) Hook. sp. pl. I p. 132 tab. 40 B; Hymenophyllu:n Gaddicr. in Voy. Uranie p. 37't tab. 5 fig. 2; Serpyllopsis antarctica v. D. B. in sched. Hab. Chili! Lecur., Peccro; Ins. Chiloe! 
Gaij, Philippl, Cun.; Ins. Falckland, Hermite! etc. J. D. IГоok; Terra Magellanica! LecilL.

114. T. Mougeoti n. sp. Fronde lanceolata pinnatifida, laciniis horizontalibus approximatis contiguisque e basi latiore oblongo-linearibus integris margine undulatis, venulis pinnatis divaricatis, cellulis opacis aegre distinguendis irregularibus inaequalibus magnis, parietibus valde flexuosis hyalinis, marginalibus parietibus rectis opacis, interaneis amorphis diffusis spissis obscure viridibus, soris primitus axillaribus, serius in latere superiore laciniarum seriatis immersis, indusio pyriformi- cylindrico margine dilatato undulato, receptaculo setaceo non exserto. Rhizoma adscendens brevissimum paleaceum, paleis aculeitormibus fuscis, ramosum, ramis glabris parceradiculosis, stipites fasciculatibasi inprimis, pariter ac rhachis venæque, paleacei vix ultra 2 centim. longi tenues teretes, frons circiter 8 centim. Jonga, 1,5-2 centim. lata plus minus obscure viridis rigidula parum diaphana.

Habitu gaudet frondis sterilis Féeae spicalae specierumque e tribu Achoma. 
nes, a quibus vero pluribus iisque gravissimis notis differt.

Ilab. Guyana Gallica! Le Prieur.

Species ignotae:

T. Guineense Sw. Syn. fil. p. 142. Guinea.

T. diffusum Bl. Enum. II. p. 225. Java.

T. Polypodioïdes L. Sp. pl. p. 1561. India orientalis.

T. dichotomum Philippl in Bot. Ztg. 1856 p. 631 et 650 . Ins. Juan Fernandez.

T. pellucidum GoLdy. Act. Acad. Leop. XIX Suppl. I p. 466. Brasilia.

T. Bryoüdes Goldm. ibid. p. 466. Brasilia.

T. Meijeniunum Pr. (Meringium) IIymen. p. 2\% tab. VIII B. Manila.

Didymoglossum Desv.

"Sori primitus terminales.

a. Costa flabellatim ramosa, venulis spuriis continuis.

1. D. punctatum (Porr.) Desv. in Ann. Soc. Linn. Paris. VI p. 330; Trichomanes PoIr. in Encl. meth. VIII. p. 6\%.

IIab. Antillarum insulae: (Martinique, Guadeloupe, Trinidad, Cuba!), Guyana! Surinam! (America centralis). 
2. D. sphenoïdes (Kze) Pr. Hym. p. 23; Trichomanes Kze in Linn. IX p. 102; T. reptans Hk. et Grev. tab. 32 (excl. Syn.)

Hab. Ins. Antillarum: (Jamaica! St. Vincent! Cuba! Guadeloupe! Barbados), Peruvia?

3. D. pusillum (Sw.) Desv. in Ann. 1. l. p. 330 ; Trichomanes Sw. Syn. p. 142. Hab. Ins. Antillarum (Jamaica! Trinidad), Brasilia! Columbia!

b. Costa pinnatim ramosa, venulis spuriis interruptis.

4. D. muscoïdes (Sw.) Desv. in Ann. l. 1. p. 330; Trichomanes Sw. Syn. p. 141; T. hymenodes Hedw. fil. gen. tab. III fig. 3.

Hab. Jamaica：Sw.

5. D. apodum (Hк. et Grev.) Trichomanes Нк. et Grev. tab. 117.

Hab. Ins. Antillarum (Barbados, Cuba), Columbia! Mexico!

6. D. Schaffneri (scnltbl.) Trichomanes Scultdu. in Linn. XXVI p. 368.

Hab. Mexico, Schiafre. (n. v.) Num differt a praecedente?

7 D. Pabstiamum (C. Mü̈L.) Trichomanes C. MüLs. in Bot. Zig. 185! p. 738. 
IIal). Brasilia (S. Catharina)! Pabst.

8. D. replans (Sw.) Pr. Ilym. p. 23; Trichomanes Sw. Syn. p. 142.

Hab. Ins. Antillarum (Jamaica! St. Domingo, Cuba!)

9. D. Kransï (Hк. et Grev.) Pr. Hym. p. 23; Trichomanes Нк. et Grev. tab. 149.

Hab. Ins. Antillarum: (Jamaica, Dominica, St. Vincent, Porto-ricco, Guadeloupe, Trinidad!) Guyana! Surinam! Columbia! Mexico?

10. D. quercifolium (Hк. et Grev.) Pr. Hym. p. 23 ; Trichomanes Hk. et Grev. tab. 115 ; T. reptans Liebm. Mex. Bregn. p. 142.

Hab. Columbia! Mexico! Ins. S. Thomas!

${ }^{*}$ Sori primitus axillares.

11. D. filicula Desv. Ann. Soc. Linn. Par. VI p. 331; Trichomanes minutulum Gadnich. in Freisc. Voy. Bot. p. 377 tab. 12 fig. 2.

Hab. Ins. Mascarenae! Madagascar! Java! Timor! Ins. Philippinae! Ins. Societatis (Borabora)! Ins. Rawack! (Bonin Sima.)

12. D. bilabiatum (N. et BL.) v. D. B. in Pl. 
Jungh. I. p. 557 (seorsim p. 13);

Trichomanes N. et BL. in Act. Acad. Leop. XI. p. 123 tab. XIII fig. 2.

Hab. Java! Sumatra? Ceylon! Mscrae, Timor! GaudiciI.

13. D. capillatum (Tascin.) Pr. Hym. p. 65; Trichomanes Tascin. Diss. p. 34 tab. I fig. 2.

Hab. Ind. orient. (m. Nilagirici !) Scrimidt ; Sumatra! Java!

14. D. undulatum Pr. Hym. p. 23 et 48.

Hab. Ins. Philippinae! Cuming.

15. D. brevipes Pr. Hym. p. 23 et 47 (1843); T. melanorrhizon Hook. sp. fil. I p. 140 (1846.)

Uab. Ins. Philippinae! Cuming.

Obs. D. decipiens Desv, in Ann. I.l. VI. p. 330 tab. VII fig. 3 quam maxime est dubium; in novo enim orbe, quantum scio, nulla species ex affinitate specierum ultimarum provenit.

Leplocionium Pr. emend.

1. L. denticulatum (Sw.) IIymenophyllum Sw. Syn. p. 148 et 375 ; Didymoglossum Pl. Jungh. I p. 560 (seorsim P. 16.) IIab. Ins. Java! Thiunb., Bu., Jungit. alii, 
2. L. Neesii (BL.) Trichomanes Bc. Enum. II [1. 226; Ifymenophylhum dicholomum N. et BL. in Act. Acad. Leop. XI ए. 127 tab. Xill fig. 4; II. mullifidum Bory in BéL. Voy. Bot. p. 82 (non Sw.)

Hab. Ins. Java! BéL., Reınw., Zoll; Ceylon! Tinvaites.

3. L. aculeatum (J. Su.) Trichomanes J. Si. in Hook. Journ. III p. 417. Hab. Ins. Philippinae! Cuming.

4. L. acanthoüdes Pl. Jungh. 1. p. 560 (Dia'y. moglossum).

Hab. Ins. Java! v. Gesk.

5. L. Braunii Pl. Jung. 1 p. 560 (Didymoglossum.)

Hab. Ins. Java! Reinw., Teysu.

6. L. holochilum Pl. Jungh. I P. 561 (Didymoglossum).

Hab. Ins. Java! Herb. A . Br.

7. L. serrulalum (Pr.) Didymoglossum Pr. Hym. p. 23 et 48 ; IIymenophyllum Smillii Iook. sp. fil. I p. 97 (p. p.) tab. 35 B; H. bivalve J. Sı. in Hoок. Journ. III p. 418 (non Sw).

Hab. Ins. Philippinae! Cunng.

8. L. affine Pl. Jungh. I P. 562 (Didymoglossitm).

Hab. Ins. Java! Teysu. 
9. L. Preshii; IIymenophyllum Smilhii Ноок.

l. 1. p. p.; Sphaerocionium bivalve Pr. in Herb. Berol. (Hym. p. 34?)

Hab. Ins. Philippinae! Meren, CuMING.

10 L. dichotomum (Cav.) Hymenophyllum Cav.

Prael. N. 688; II. plicatum Kaulf.

Enum. p. 268.

Hab. Chili! Lecril., GaY, alii.

11. L. Magellanicum (Dêsv.) Didymoglossum Desv. in Act. Soc. Linn.Par. VI.p. 331; Plychophyllum KL. in Linn. XVIII. p. 533. Hab. Terra Magellani, Chili! LEcIL., Pinlispri.

12. L. dentatum (Cav.) Itymenophyllum Cav. Prael. N. 687 .

Hab. Ins. S. Carlos de Chiloe! NEÉ.

13. L. tortuosum (Нк. et Grev.) IIymenoplyllum IIк. et Grev. tab. 129; Mijrmecoslylum Pr. Hymen. p. 27 tab. 10 A. Hab. America antarctica, Menz., J.

D. Hoок.! Lechl.! alii.

14. L. Seselifolium Pr. Hymen. p. 33 et 52. Hab. Chili, Cumung (n. v.)

15. L. attemuatum (Ноо.) IIymenophyllum Ноок. sp. fil. I. p. 99 tab. 36. A. Hab. Brasilia, Galedn.; (n. v.) Obs. Didijmorglosso et Ilymenophyllo 
genus intermedium, quod, si ulterior observatio confirmaverit, pluribus $\mathrm{Hy}^{\mathrm{y}}$ menophylli speciebus ditatum iri existimo.

B. HyienophyLLö̈deAE.

Hymenoglossum $\mathbf{P}_{\mathbf{R}}$.

1. II. cruentum (Gav.) Pr. Hym. p. 35; Hymenophyllum Cav. Prael. N. 684.

Hab. Chili! Née, Lecnl., Gay, alii; (Mexico? Hatenke).

Hymenophyllum Su.

A. Frons glabra,

a. margine integro. a. Frons plana.

† Venae a basi inde furcato-dichotomae.

1. H. marginatum Hk. et Grev. tab. 37.

Hab. Nova Hollandia, Fraser (n. vo)

2. II. brevifrons Kze. Farrnkr. 1 p. 236 tab. 96 fig. 2.

Hab. Guyana Gallica! Le Prieur.

3. H. palmatum; Trichomanes flabellatum BorY in Bèz. Voy. Bot p. 77.

Hab. Ins. Mascarenae! Bonx, Bèc., Borvis. 
it venae pinnatim ramosae.

4. II. abruptum Hook. sp. fil. I p. 88 tab. $31 \mathrm{~B}$.

Hab. (Jamaica), Cuba! Wrigitr.

5. II. brevistipes Lieb. Mex. Bregn. p. 138. Hab. (Mexico); Cuba! Wrignt; Caraccas! Funk et Schum.

6. II. asplenioïdes Sw. Syn. p. 145.

Hab. Jamaica! Mexico! (Guyana anglica, Brasilia?)

7. II. capillaceum Rохв. Ноок. sp. fil. I p. 109 tab. 38 B; II. infortunatum Bony in Voy. Uran. Bot. p. 284 tab. 38 fig. 3.

Hab. Ins. S'. Helena, Menz.! J. D. HOOK! M'RAE! alii.

8. II. Fumarioïdes Bony in Willd. Sp. pl. V p. 526.

Hab. Ins. Mascarenae, Bory!

9. II. gracile Bory in Willd. sp. pl. V p. 527.

Hab. Ins. Mascarenae, Bory! HowBRon! BoIvin! alii.

10. II. Natalense n. sp. Fronde lanceolata vel lineari basi pinnata caeterum bipinnatifida, laciniis primariis erecto-patulis imbricatis e basi cuneata acuta lanceolato-oblongis pimnatifidis, se- 
cundariis contiguis strictiusculis obovatis cuneatisque simplicibus furcatisve, lacinulis strictis latis apice emarginatis, cellulis magnis opacis parumper elongatis acutangulis chlorophyllosis, globulis laete viridibus diffusis, marginalibus duplo fere minoribus non elongatis, parietibus hyalinis incrassatis hic illic leviter undulatis, soris praemagnis rhomboïdeo-oblongis basi conicis antice bilobis, lobis vix $1 / 2$ sorum aequantibus integris leviterve erosis, receptaculo brevi incluso. Rhizoma ultra setaceum horizontale, stipes teres setaceus usque $\mathbf{7}$ centim., frons $10-12$ centim. longa, $11 / 2-2$ centim. lata fusco-olivacea parum diaphana debilis.

Hab. Port Natal, Gueinzius ! loco non designato, DrèGe! Klynrivier, Zeijher! Gnadenthal, Bredtel!

Obs. Vix dubie huc citandum $I I$. Fumarioïdes auctorum de filicibus Capensibus (Schltdo. Adumbr. pl. Cap. p. 56 (excl. pl. Brasil.; tab. 35 citata numquam prodiit); Kunze Pl. acotyl. Afr. p. 75; etc.), quae vero species omnibus omnino notis a nostra rece- 
dit. Specimina ZfiJieri el breutelil in schedulis nomine $\mathrm{H}$. Zexireri distinxi, quippe quae habitu et divisione frondis, soris duplo minoribus, inprimis vero contextu frondis tantopere singulari differunt, ut normalis an sit dubitem. Propterea hic indicasse sufficiat dum ulterior observatio quid pro vero habendum sit, docuerit.

11. I. rarum R. Br. Prodr. Fl. Nov. Holl. p. 159 (seorsim p. 808); H. semibivalve Нк. et Grev. tab. 83.

Hab. (Tasmannia), Nov. Zeelandia, J. D. Hоoк :

12. I. flabellatum Labill. Nov. Holl. pl. spec. II. p. 101 tab. 250 fig. 1; $I$. nitens R. BR. Prodr. p. 159 (seorsim p. 808.)

Hab. Tasmannia! van Diemensland! Australia felix! etc.

13. H. Hookeri Bony in Bèz. Voy. Bot. p. 84; H. nitens Hк. et Grev. tab. 197 (non R. Br.); $H$. /labellatum $\beta$ Hook. sp. fil. I p. 111.

Hab. Nova Zeelandia! Ins. Auckland! (Java.) 
14. I. polyanthos Sw. Syn p. 149.

Hab. Jamaica! Sw. Columbia! Morru z

15. H. Grevilleanum Pr. Hymen. p. 32 II. polyan-

thos HK. et Grev. tab. 128 (non Sw.)

Hab. Jamaica! ( $\mathbf{S}^{\mathbf{t}}$. Vincent.)

16. H. Schomburgkii Pr. Hymen. p. 32; Trichomanes clavatum Kavlf. in Weig. Exsicc. Surin.; II. polyanthos Kze in Linn. XXI p. 2\%0; II. clavalum Splits. in Tijdschr. voor Nat. Gesch. VII p. 437.

Hab. Guyana Batava! Anglica!

17. H. Martinicense. H. decurrens $\beta$. Sieberi Pr. Hymen. p. 32.

Hab. Martinique! Sıeb. ; Guyana Gallica! Le Prieur.

18. II. Poeppigianum Pr. Hymen. p. 32 et 54; H. clavatum Kze in Linn. IX p. 109; II. polyanthos Metrex. in Fil. Lechl. p. 26.

Hab. Peruvia! Lechl.; (Guyana Anglica).

19. H. axillare Sw. Syn. p. 148.

Hab. Jamaica! Sw.

20. H. millefolum Schutdu. in Linn. V p. 620 ;

H. polyanthos Pr. Hymen. p. 32.

(non Sw.)

Hab. Mexico. (n. v.)

21. H. abietinum Нk. et Grev. tab 127. 
Hab. Peruvia , Jameson, Lechl. ! Couthoux!

22. I. cunealum KzE Anal. pterid. p. 50 (1837); H. Cumingii Pr. Hymen. p. 32 et 56 tab 11 A (1843.)

Hab. Ins. Juan Fernaudez. (n. v.) 23. H. australe Willd. Sp, pl. V p. 527; $\boldsymbol{H}$. polyanthos Labill. (teste spec. auth.)

Hab. van Diemensland! Taïti! Planch, et Vieill.

24. II. Blumeanum Spr. S. V. IV p. 131; H. pectinatum $\mathbf{N}$. et BL. in Nov. Act. Leop. XI p. 124 tab. XII fig. 5 (non CAv.)

Hab. Java! Herb. AL. Br.; Sumatra! Tersu.

Obs. Speciminibus incompletis deceptus $\boldsymbol{H}$. lineari ante habui affine. Meliora nunc edoctus non alienum esse video a speciebus, seriem $\boldsymbol{H}$. polyanthi in regionibus Indo-australibus repetentibus, imo praecedenti, quocum structura omnino convenit, olim subjungendum esse facile crediderim.

25. H. integrum v. D. B. in Pl. Jungh. I p. 563 (seorsim p. 19.); $H$ emarginatum N. et BL. l. l. p. 127 tab. XI fig. 5 (non Sw.)

Hab. Java! Be. 
26. H. pycnocarpum v. D. B. in Pl. Jungh. I.

p. 564 (seorsim p. 20.)

Hab.Java! Herb. Ac. Br, (Gedé) v.Gesk

27. II. coloralum AL. BR. in Pl. Jungh. 1 p. 565

(seorsim p. 21.)

Hab. Java! Herb. Ac. Br.

28. II. tenellum Dos. Prodr. Fl. Nepal. p. 12;

Ноок. sp. fil. I p. 112.

Hab. Nepal! Wall. (spec. ex dono Lambert in Herb. Sond.)

29. H. Wrightii $\mathrm{n}$. sp. Fronde late oblonga pinnatifida, laciniis patulis contiguis 1-2 dich otomis dimidiatis (?), lacinulis latiusculis abbreviatis, cellulis opacis firmis rubro-fuscis magnis (imo maximis) elongato-hexaëdris acutangulis globulosis, globulis inaequalibus confertissimis diffusis, parietibus hyalinis tenuibus rectis, cellularum marginalium minute et obtuse crenulatis, soris in laciniis axillaribus reflexis lacinulâ latioribus e basi conica immersa bilobis, lobis semicircularibus integris, receptaculo brevi. Rhizoma horizontale ramosum setaceum paleis piliformibus elongatis crispulis parce tomentosum; stipes mediotenus ala rhacheos descendente anguste marginatus vix ultra 7 millim. longus, frons 16 
millim. longa, 10 millim. lata opaca firmiuscula rubro-fuscidula.

Hab. Japonia! (Hakodadi), Wrigir. 30. H. paniculiflorum Pr. Hymen. p. 32 et 55. Hab. Ins. Philippinae, Cusing. (n. v.)

31. II. clavatum Sw. Syn. p. 149; H. fraternum? Pr. Hymen. p. 32 et 54 tab. 12 B. Hab. Jamaica! Sw.; Porto-ricco! Sghwanecke.

32. H. protrusum Ноок. sp. fil. I p. 104 tab. 37 B; H. clavatum Sw. (p. p.) teste spec. auth.

Hab. Mexico! Sincl.; Jamaica! Sw.

33. I. ferax. n. sp. Fronde lanceolata tripinnatifida, laciniis primariis oblongolanceolatis mediiscontiguis, infimis summisque remotiusculis, patulis apice arrecto bipinnatifidis, secundariis oblongis obovatisve contiguis patulis vel subdivaricatis pinnatifidis, tertiariis pinnatifidis apicalibus simplicibus, lacinulis simplicibus dichotomisve brevibus latis apice integro, cellulis rubro-fuscis validis opacis mediocribus subaequalibus parum elongatis obtusangulis, interaneis amorphis spissis diffusis, parietibus vix conspicuis flavescentibus rectis, marginalium cel- 
lularum incrassatis crenulatis, soris magnis numerosis turgidis in lacinulis tertiariis lateralibus e basi conica bilobis, lobis sori $2 / 3$ aequantibus oblongis margine eroso, receptaculo brevi incluso. Khizoma -? stipes teres setaceus debilis 7 centim. et ultra longus, frons 2 decim. longa, 3-4 centim. ląta opaca fusco-olivacea firmiuscula pendula(?)

Hab. Venezuela! Furck et Scillim (N. 1578.)

34. II. Jalappense Scrltod. in Linn. V p. 619; II. polyanthos KzE in Linn. XVIII p. 352 .

Hab. Mexico, Scinede! Scinaffner! alii.

35. II. Kohautianum Pr Hymen. p. 32 et 56. Hab. Martinique! Sieb.; Guadeloupe!

L' Hermin.

36. II. recurvum Gaudich. in Voy. Uran. Bot. p. 576.

Hab. Ins. Sandwich! Gaudicir.

37. II. acrosorum v. D. B. in Pl. Jungh. I p. 564 (seorsim p. 20.)

Hab. Java! v. Gesk.

38. II. Aucklandicum. H. crispatum $\delta$ Tasmanicum Ноок. sp. fil. I p. 105 excl. Syn. R. Br. et? Colenso). 
Hab. Tasmania! Ins. Auckland! J. D. Hook. in Herb. Sond.

Obs. Synonymon Hookerianum citare jubent specimina viri clarissimi in Herb. de Vriese. Caeteroquin speciem, quam equidem ad specimina Aucklandica (in Herb. Sonder) determinavi, nondum descriptam credidissem, quandoquidem $\boldsymbol{I}$. flabellatum R. Br., ab illustri auctore synonymon citatum, toto coelo diversum esse e specimine authentico compertus sim. Plantam, a Colesso nomine $I$. atrovirentis descriptam identicam esse cum illo $\boldsymbol{H}$. flabellato suspicor, at specimine non viso judicium ferre nequeo.

39. H. caudiculatum MarT. Icon. sel. Brasil. p. 102 tab. 67.

Hab. Brasilia, MArt! Beyr.! alii.

40. II. caudatum Sphaerocionium productum PR. Hymen. p. 35 et $61 ; \boldsymbol{I}$. fuciforme Metten. in Fil. Lechl. p. 26 ; H.caudiculalum $\beta$. Ноок. sp. fil. I p. 102. Hab. Chili, Gay! Lecul.! (Ins. Chiloe).

41. H. fuciforme Sw. Syn. p. 148.

Hab. Chili, LechL.! (Ins Chiloe, Juan Fernandez). 
42. H. Junghuhnii v. D. B. in Pl. Jungh. I. p. 570 (seorsim p. 26); H. dilatatum Be. Enum. II p. 221. (non Sw).

Hab. Java, Resww.! JungiI.! Zol...!

43. II. macrocarpum (PR.) Sphaerocionium Pr.

Hymen. p. 35 et 61.

Hab. Ins. Philippinae! Cunivg.

44. H. badium Нк. et Grev. tab. 76.

Hab. (India orientalis), walc. (n. v.).

45. H. Cumingii. Sphaerocionium badium Pr. Hymen. p. 35. (non Hk. et Grev.)

Hab. Ins. Philippinae! Cusstrg.

Obs. II. badium secundum descriptionem et iconem) differt: fronde e basi obtuso elliptica, laciniis primariis brevibus, soris in hisce axillaribus solitariis, indusii lobis integerrimis, colore balio, etc.

46. II. sororium (Рr.) Leptocionium Pr. Epimel. p. 21 tab. 11.

Hab. (Nova Zelandia), Java! v. Gesk., JUNGiI.

17. H. leptodictyon C. Mü̈L. in Bot. Ztg. 185' p. $73 \%$.

Hab. Java! Zoll., v. GeSk.

48. H. demissum (Fonst. Sw. Syn p. 147 et 37ł; Trichomanes Fonst. Prodr. p. $85 \mathrm{~N}$. 468.

Hab. (Ins. Inaris pacifici, Fonst.), 
Philippinae! Coming, Nova Zelandia, Sinclair! J. D. Ноoк.! alii.

49. I. eximium KzE in Bot. Ztg. 1846 p. 478 (descr. ad specimina incompleta); $\boldsymbol{H}$. inclinatum v. D. B. in P]. Jungh. I p. 570 (seorsim p. 26.) Hab. Java, ZoLL! v. GeSK.!

Species ignotae :

II. emarginalum Sw. Syn. p. 148 et 377. Java, Truns.

II. imbricalum BL. Enum. p. 220. Java.

1I. ramosissimum Hayilt, in Don Prodr. Fl. Nepal. p. 12. Nepal.

$\beta$. Frons squarrosa crispa vel undulata.

50 II. sanguinolentum Sw. Syn. p. 529.

Hab. Nova Zeelandia Sincl! Cun!

J. D. Ноок! Mosm! alii.

51 H. pulcherrimum CoL Hook. sp. fil. I. p. 103 tab. 37 A.

Hab Nova Zeelandia, Cor. (n. v.)

52 H. imbricalum CoL. (in Tasm. Phil. Journ.) H. $\operatorname{rarum} \beta$. Ноок. sp. fil. I p. 101. Hab. Nova Zeelandia, J. D. Ноок.! Java, Teysu.! (Chiloe? Terra del fuego?

Obs. Cum II. raro R. BR. ne habitu quidem convenit. Nomen valde aptum mutandum erit si homonymam 
speciem Blumeanam, nunc Bolanicis ignotam, aliquando inter species cognitas recipere licebit.

53. II. tabulare. II. rarum Kze Farrnkr. I p' 234 tab. 96 fig. 1 (non R. Br).

Hab. Africa australis (Tafelberg, Duivelsberg, Hollandsberg) ECKL! ZEYHER！ DrèGe！

54. II. apicale H. axillare $\mathrm{Hk}$. et Grev. tab 124. (non Sw.).

Hab. Jamaica! (S'. Vincent.)

Obs. Differt ab II. axillari Sw, habitu frondis et divisione, stipite et rhachi inferne nuda filiformibus, soris lacinula duplo angustioribus e basi cuneata oblongo-trapezoïdeis terminalibus, cellularum denique formaet magnitudine.

55. H. nigricans (PR.) Kr. in Linn. XVIII p. 536 ; I. polyanthos $\gamma$. Ноок. sp. fil. I. p 107 (p. p.)

Hab. Columbia ! Monitz; Peruvia, Domb. MATtil.!

56. H. myriocarpum Ноок. sp. fil. I p 106. tab. $37 \mathrm{D}$.

Hab. (Columbia, Hartw.) Caraccas! Funk et Schlin, Monitz?

57 II. Anaimum n. sp. Differt a praecedentibus: stipite debili, pariter ac rhachis in- 
ferne, non alato, fronde tenui flaccida, ab II. myriocarpo insuper: laciniis primariis elongatis apice arrectis, lacinulis latioribus et longioribus, soris oblongis obovatisve, indusii lobis emarginate-crenatis, contextu frondis; $a b$ I. nigricante: laciniis secundariis elongatis, lacinulis plus quam duplo latioribus apice integris, soris duplo majoribus, cellularum forma et magnitudine.

Hab. Andes Quitenses! Couthouy.

58 H. Ricciaefolium Bory in Willd. sp. pl. V p. 531; Adiantum tenellum $J_{A C Q}$. collect. III p. 287 tab 21 fig. 3 (rudis sed bona.)

Hab. Ins. Bourbon! Bork, Borvin; I'adagascar! Goudeot.

59. H. crispatum Wall. Hook. sp. fil. I p. 105 ? (excl. saltem $\delta$ ); Hк. et Grev. tab 77. Hab. Nepal, Wall. (n. v.)

60. II. erosum BL. Enum. II. p. 221.

Hab. Java, Bc.!

61. II. fimbriatum J. Sm. in Hook. Journ. III. p. 418.

Hab. Ins. Philippinae, Cur.! Java, v. GESK! 
62. II. Jacanicum Spr. S. V. IV p. 132; II. crispum $\mathrm{X}$. el Bl. in Act. Acad. Leop. XI p. 128 tab. XIV fig. 1.

Hab. (India orientalis), Ceylon!

Gardx. Java! Bu., Zouc., alii.

63 II. micranthum v. D. B. in Plant. Jungh.

I p. 566 (seorsim p. 22).

Hab. Java, v. Gesk.!

6.) H. Reinwardti v. D. B. in Plant. Jungh.

1 p. 567 (seorsim p. 23.); H. dichotomum BL. Enum. II p. 222 (non Cav., nec N. ab Es.)

Hab. Java, Reivw.!

65. H. produclum KzE in Bot. Ztg. 1848 p. 305.

Hab. Java, ZoLl! v. GeKs.! alii.

66. II. flexuosum Curs, in Hoor. Bot. Mag.

Compan. II. p. 369.

Hab. Nova Zeelandia, Cuns, F.

Muller! Sixclair! alii.

67. H. Tasmannicum H. flabellalum R. BR. Prodr. p. 159 (seorsim p. 808 (non Labill.) H. crispalum $\delta$ Ноок. sp. fil. I p. 105 (p. p.)

Hab. van Diemensland! R. Bß.

$$
\text { Species ignota: }
$$

II. daedaleum BL. Enum. II p. 220. Java. 
68. 11. undulatum Sw. Syn. p. 148; Hedw. gen. fil. tab. XIX (non XI).

Hab Jamaica! Sw.; Peruvia! Matтh.

69 II. flaccidum. H. undulatum Hedw. gen. fil. tab. XI; H. myriocarpum? Metten. in Fil. Lechl. p. 26. (non Ноок.)

Hab. Jamaica! Cuba Wright; Columbia! Monıтz; Andes Quitenses: Saettone; Mexico! Schaffn; Peruvia! LECHL.

70. H. crispum HBK. Nov. Gen. I. p. 258 ; Kunth Syn. p. 90. II. undulatum MetTEN. in Fil. Lechl. p. 26 (non Sw.)

Hab. (Columbia, Humb. , Bonpl.);

Peruvia, Lecil.

71 H. reniforme Ноoк. Sp. fil. I p. 110 tab. $38 \mathrm{C}$.

Hab Peruvia, Matth. (n. v.)

72. H. convolutum n. sp. Fronde lineari bipinnata, pinnis e basi cuneata oblongis vel oblongo-linearibus interdum ramulos plus minus elongatos simulantibus aequidistantibus erecto-patulis in fronde fertili contiguis, in sterili remotis pinnatis, pinnulis approximatis patulis obovatis vel subflabellatim cuneatis simplicibus vel $1-3$ furcatis, lacinulis linearibus antice latioribus emarginatis longitudinaliter 
convoluto-undulatis, cellulis teneris hy. alino-diaphanis (subtiliter punctulatis) mediocribus, imomagnis, subaequalibus regulariter subelongato-hexaëdrisacutangulis, interaneis frustuloso-nebulosisparietalibus (raro diffusis) e rubro fuscidulis, parietibus hyalinis rectis tenuibus, soris in lacinulis terminalibus parvis turgidis, indusio e basi rotundato-cuneata bilobo, lobis $\frac{3}{4}$ sori partes aequantibus suborbicularibus antice denticulatis, receptaculo brevi. Rhizoma ultra setaceum, stipes setaceus (pariter ac rhachis) teres fusco-ater circiter 5 centim. longus, frons $1-11 / 2$ decim. longa, $15 \mathrm{mil}-$ lim. lata debilis diaphana e pallide badio rubro-fusca.

Hab. Andes Quitenses! Couthouy, JAMESON.

Species ignota:

H. Endiviaefolium Desv. Ann. soc. Linn. Par. VI p. 334. Peruvia.

b. margine dentato.

๙. Indusium integrum.

73. II. Wilsoni Ноок. Brit. Fl. I. p. 446; Engl. Bot. Suppl. I tab. 2686. 
Hab. Brittannia! (inprimis Hibernia et Scotia); Ins Far! Norvegia austr. et occid! (Saxonia)?

74 H. megachilum Pr. Epimel. p. 22 tab. 8 B. Hab. Brasilia, Gardn. (n. v.)

$75 \mathrm{H}$. unilaterale Bony in WiLLd. sp. pl. V. p. 521.

Hab. Ins. Bourbon, Bony!

76 H. Meyeri Pr. Hymen. p. 31 et 50.

Hab. Africa australis, Drège! LeIBOLD! Natal GueINzius!

77 II. cypressiforme Labilx. Nov. Holl. pl. spec. p. 102. tab. 250 fig. 2; I. Tunbridgense $\beta$ Ноок. sp. fil. I p. 95 (p. p.)

Hab. Nova Zeelandia! LABıLL.

78. II. Menziesï Pr. Hymen. p. 31 et 51; H. Tumbridgense Metren. Fil. Lechl.p. 26.

Hab. America antarctica; Terra Magellani, Lecul! Hermite Isl., Cape Horn, J. D. Ноок! (Staatcnland, Menz.)

79. H. secundum IIK. et Gnev. tab. 133.

Hab. America australis et antarctica;

Chili! Puccio; Terra Magellani! Lecrul.; Hermite Isl., Cape Horn, J. D. Ноок! (Staatenland, Mexz.)

80. H. serra Pr. Hymen. p. 32 et 53 tab. XI B; II. secundum Metren Fil. Lechl: p. 26 (p. p. 
Hal. (Chili, Cumng); Valdivia! PuL.IPPI.

81. H. pectinatum Cav. Prael N. 685.

Hab. Chili, Gaij! Lecill.! Puccio! alii.

82. I. Bridgesï Ноок. sp. fil. I p. 97 tab. 35 C. Hab. Chili, Bridges, Cuming, PuiLIPP1 !

83. II. Bibraiamum Stunм in Flora 185.3 p. 361 .

Hab. Chili, Bibra. (n. v.)

84. H. bivalve (Fonst.) Sw. Syn. p. 146 et 372;

Trichomanes Fonst. Prodr. p. 460.

Hab. (Nova Zeelandia, Forst., Cor.); Ins. Auckland ! J. D. Ноок.

$\beta$ indusium dentatum.

85. H. Tunbridgense Sx. Fl. Britt. III p. 1141.

Hab. Brittannia! Gallia occidentalis ! (Italia superior), Corsica! Tyrolis australis! (Saxonia ?)

86. H. Drègeanum Pr. Hymen. p. 32 el 52 ; H. Tunbridgense $\beta$ pedunculatum KzE. in Pl. acotyl. Afr. austr. p. 74.

Hab. Africa australis! Drége, EcKLON, alii.

87. H. minimum Ricr. Fl. Nor. Zeel. p. 91 tab. 14. fig. 2; H. antarcticum $\mathrm{PR}$. 
Hymen. p. 31 et 50 tab. $12 \mathrm{~A}$ ?

Hab. Nova Zeelandia, Sıєв.! J. D.

Ноок.! Mossu.! alii.

88. $\boldsymbol{H}$. asperulum Kze in Linn. IX p. $109 ; \boldsymbol{H}$.

Tunbridgense Metten. Fil. Lechl. p. 26 (p. p.)

Hab. Chili, Poepp., Philuppı!

Species ignota:

H. Thunbergii (Ecks.) Pr. Hymen. p. 32.

Cap. bon. spei.

89. H. fucoüdes Sw. Syn. p. 146 ; Trichomanes

Hedw. gen. fil. tab. VIII.

Hab. Jamaica! Columbia (Venezuela!

Nova Grenada!), (Mexico, Peruvia

Brasilia?)

90. $H$. pedicellatum KzE in Linn. XX p. 439 .

Hab. Columbia, Moritz! Funk et Schlin!

91. H. Peruvianum Hк. et Grev. tab. 208.

Hab. Columbia (Andes Quitenses,

James.！Saettone! Couthouis! Nova Grenada, SciıLiм)?.

92. H. spmulosum H. B. K. Nov. gen. I p. 26 ; KтII. Syn. pl. orb. nov. p. 90 ; Leptocionium fucoïdes $\mathrm{Kl}$. in Linn. XVIII p. 533. 
Liab. Columbia, IIunrs, et Boxpr.,, Monitz! Fuxk et Scillim!

Obs. Species hae quatuor licet parvitate non peccent, varie confusae sunt. II. pedicellati nomen e schedulis Kunzesnis edens, describendi curam non prima vice aliis commode relinquens, Cl. Kьотsci distinguendum id ab II. fucoïde monet: indusii valvulis ovato-lanceolatis, margine inaequaliter serrato-dentatis; quasi hoc a specie Sivartziana alienum esset. II. Peruvianum, etiamsi pinnatum dicat Cl. Нooker, rhachi inferne anguste alata gaudere videtur situque et directione sororum omnino cum II. fucoïde convenit, cujus quippe sori vetusti demum reflectuntur. Utroque insignius II. spinulosum. Quae vidi adque auctorum sententiam determinavi specimina contextu frondis duplicem typum monstrant; alteram cellulis firmis opacis magnis parietibus rectis, teneris hyalinis facile maximis parietibus crenulatis alteram.

93. H. blepharodes Pr. Hymen. p. 32 et 51. Hab. Martinique! Guadeloupe! 
94. II. Jamesoni Hook. sp. fil. I p. 96 tab $35 \mathrm{~A}$.

Hab. Andes Quitenses! JaMeson.

95. H. cristatum Hк. et Grev, tab. 148.

Hab. Andes Quitenses, JaMeson. (n. v.)

96. II. multifidum (Fonst.) Sw. Syn. p. 149 et 378; Trichomanes Fonst. Prodr. p. 85 N. 473.

Hab. (Ins. maris pacifici, Fonst.); Nov. Zeelandia, Lyall! J. D. HooKEn! alii.

\section{B. Frons vestita,}

a. pendula (stipite plerumque debili).

$\alpha$. Venae venulaeque alato-cristatae.

97. H. sericeum Sw. Fl. Ind. occid. III p. 1745; Plum. Foug. p. 56 tab. 73; Hedw. gen. fil. tab. VII.

Hab. Ins. Antillarum (Jamaica! Cuba! Guadeloupe! Martinique, Trinidad!)

98. II. plumosum KLF. Enum. p. 267; Sphaerocionium aureum Pı. Hymen. p. 3/4 et 57 .

Hab. Brasilia! Columbia! Peruvia!

99. II. tomentosum KzE Linn. IX p. 107; Farmks. I p. 160 tab. 69; H. seri- 
ceum Metten. in Fil. Lechl. p. 25 (non Sw.)

Hał. Peruvia, Pörp.! Lechl.!

Species ignotae:

II. Myramidatum Desr. in Ann. Soc. Limn. Paris. VI p. 332. America tropica.

11. lobalo-alatum KL. in Limn. XX p. 438. Peruvia.

$\beta$. Venae venulaeque nudae.

100. H. hirsutum Sw. Fl. Ind. occid. III p. 1746; Hedw. gen. fil. tab. $\mathbf{X}$.

Hab. Jamaica! Cuba! Columbia! Mexico?

101. H. latifrons n. sp. Fronde oblonga rel lanceolato-oblonga pinnatifida, laciniis inferioribus obcuneatis 1 -3dichotomis, reliquis oblongo-linearibus furcatis simplicibusque patulis subaequidis tantibus, lacinulis margine leviter undulatis apice retusis, venulis gracilibusprope apicem desinentibus, pilis frondis marginem et laminam, pariter ac rhachidem, venas venulasque dense obtegentibus debilibus elongatis apice in radios 2-4 erectiusculos divisis, cellulis hyalinis maximis elongatis regularibus acutangulis, parietilus 
parum diaphanis rectis tenuibus, interaneis parietalibus amorphis fuscidulis, marginalibus magis elongatis subtetraëdris, soris parvis in lacinulis terminalibus hisque angustioribus, indusio e basi conico-rotundata bilobo lobissuborbicularibus transversim latioribus undulatis integris dense ciliatis, receptaculo setaceo brevi.

Rhizoma horizontale setaceum repens, pariter ac stipes brevissimus (1-1,5 centim.) teres capillaris, hirsutum, frons $4-5,5$ centim. longa, $2-2,5$ centim. lata diaphana tenera olivaceo-fuscidula, lacinulae 3 millim. latae.

Hab. Guadeloupe! Pernott., L'Hermin. 102. II. venustum Desv. in Ann. Soc. Linn. Paris. VI p. 332 ; $H$. hirsulum Raddr Fil. Brasil. p. 19 tab. 79 fig. 3 (non Sw.); II. Raddianum MüLL. in Bot. Ztg. 1854 p. 722.

Hab. Brasilia, Raddi (n. v.)

103. H. pulchellum Schltdol. in Linn. V p. 618.

Hab. Mexico! Scmede, Scuaffn.

10\%. II. elegantulum. II. pulchellum Ноок. sp. fil. I pag. 91 ? tab. $33 \mathrm{~A}$ ! (non SchldL) IIab. Andes Quitenses! James., SaETTONe. 
105. II. procerum. H. pulchellum Mlistes. in Fil. Lechl.p. 25 (non Scilltul., nec IIook).

Hab. Peruvia! Lecill.; Columbia! Janes., RéuiJ.

106. H. lineare Sw. Fl. Ind. occid. III p. 17'9;

H. elegans SPR. S. V. IV p. 133;

II. trifidum Нк. et Grev. tab. 196.

Hab. Brasilia! Peruvia! (Columbia), Nova Grenada!

107. I. Crugeri MüLs. in Bot. ZIg. $185 \%$ p. 722 .

Hab. Trinidad! Crüger; Guadeloupe! L'Heruiv.

108. H. interruptum Kze. Anal. p. 48 tab. 30.

Hab. Peruvia, Pöppıg (n. v.)

109. $H$. aequabile KzE. sec. KL. in Linn. XX p. 138 (a neutro descriptum!)

Mab. Columbia, (Venezuela, Moritz! Fuxk et Scilim! Nova Grenada, Scrlin!)? Peruvia, Lecul.!

Obs. Speciene differat ab antecedente, me judice, denuo inquirendum est. II. interruptum coll. Poeppigiana, quod vidi, evidenter pertinet ad $I$. lomentosum Kze. Specimina Peruviana a Lechlero collecta e contextu frondis huc referenda sunt; sumt auten sterilia ideoque non tute determinauda. 
110. II. pendulum Bory in Bèz. Voy. Ind, or. Bot. p. 81 tab. 8 fig. 2.

Hab. Ins. Bourbon, Bonis! Boivis! de Montroison! alii.

Species ignotae:

II. mudum (PoIr.) Desv. Ann. Soc. Linn. Par. VI p. 332. Guadeloupe.

II. capillare Desv. ibid. p. 333. Ins. Tristan d'Acunha.

b. erecla (stipite firmiore.)

111. H. Organense Ноок. sp. fil. I p. 90 tab. $32 \mathrm{~B} ;$ II. Beïrichianum KzE in Linn. IX p. 108 (quo ad pl, Brasil.)

Hab. Brasilia, Gandn., Bexn.! Columbia (Venezuela! Monitz, N. Gre. nada! Scilım.)

112. H. Beyrichianum Kze in Linn. IX p. $\mathbf{1 0 8}$ (quo ad pl. Peruv.)

Hab. Peruvia, Poepp. (n. v.)

113. H. Ruizianum KL. (Sphaerocionium) Linn. XVIII p. 535.

Hab. Peruvia, Rorz (n. v.)

114. II. Lindeni Ноoк. sp. fil. I p. 94 tab. $34 \mathrm{C}$; II. spectabile Monitz in Coll. Fil. Columb.

Hab. Venezuela, Linden, Monitz! 
115. H. valvatum Нk. et Grev. tab. 219.

Hab. Andes Columbicae, Janes.! Peruvia, Lecill.

116. II. Francarillei n. sp. Fronde oblongolanceolata apice producta bipinnatifida, laciniis primariis e basi lata triangularibus ovatisve imbricatis contiguisque subhorizontalibus, imis deflexis, pinnatifidis, secundariis obcuneatis remotiusculis patulis infimis $2-3 \mathrm{di}$ chotomis, reliquis simplicibus furcatisve, lacinulis elongatis undulatis margine integro dense pilis simplicibus hyalinis obsesso, apice attenuatorotundato, sinubus cunctis latis obtusis, venis venulisque tenuibus fuscis, pariter ac rhachis, parce pilosis cellulis parum diaphanis magnis maximisque inaequalibus subregularibus elongatis, parietibus subdiaphanis rectis parum incrassatis, interaneis amorphis globulosisve primum diffusis serius parietalibus, globulis minutis flavo-fuscis, marginem versus minoribus latitudine usque $2^{1} / 2$ longioribus, soris in lacinulis terminalibus, indusio e basi elongato-conica immersa oblongo bilobo, lobis anlice eroso-denticulatis ciliatis nudisve. 
1hizoma ultra setaceum dense ramulosum paleaceo-hirsutum, stipes teres, basi excepta, glaber obscure fuscus 5-7 centim. longus, frons 10-14 centim. longa, 4-5 lata olivacea diaphana undulato-squarrosula rigidiuscula.

H. valcatum si non erraverim in determinandis speciminibus Peruvianis, differt: fronde e basi lata ovata, lacinulis latioribus sinubus angustis acutis interstinctis minus undulato-crispis, cellulis majoribus interaneis amoene viridi-chlorophyllosis, marginalibus opacis (lacinulis hinc sub lente marginatis), soris majoribus etc.

Uab. Guadeloupe! Perrottet; Martinique! Bélanger.

117. II. Plumieri IIook. et Grev. tab. 123 (excl. Syn. Plum.)

IIab. Andes Columbianae, Jaues., Couthouls!

118. II. trapezoïdale Lieвm. Mex. Bresn. p. 141. Hab. Mexico, Lieba. (n. v.)

119. II. hirtellum Sw. Syn. p. 149.

Hab. Jamaica! St Vincent! Guadcloupe! Mexico!

120. II. crispatulum. II. hirtellum METTEN. in Fil. Lechl. p. 25 (uon Sw.)

IIał. Peruvia, Lechl.! 
Obs. H. valvato magis quam $H$. hirtello affine, quorum hoc quidem differt: fronde lanceolata, laciniis primariis patulis, secundariis simplicibus furcatisve, lacinulis duplo fere latioribus, rhachi plana, cellulis validis opacis majoribus, interaneis (quatenus globulosis) minutis diffusis aureis; illud vero: fronde subcordato-ovata, laciniis primariis latioribus imbricatis, lacinulis plus quam duplo latioribus, rhachi plana, stipite terete, cellulis majoribus, parietibus rectis, interaneis distincte et pulchre viridi-globulosis, etc.

121. II. ciliatum Sw Fl. Ind occid. III. p. 1753; Syn. p. 147; Plum. Foug. p. 73 tab. 50 fig. D.; Sphaerocionium vestitum Pr. Hym. p. 34 et 58.

Hab. Jamaica! Guadeloupe! Martinique! Mexico?

122. H. remolum. II. ciliatum Нк. et Grev, tab. 35 (non Sw.); Sphaerocionium Grevilleanum $\mathrm{Pr}$. Hymen. p. 3 r ;? II. Borijanum MetTen in Fil. Lechl. p. 25. Hab. (Ins. S. Vincent,) Columbia; Moritz; Guyana Gallica! LePreen; Brasilia! Séllow, Langso.; Peruvia? LECIIL. 
123. H. Surinamense. Sphaerocionium ciliatum Pr. Hymen. p. 34.

Hab. Surinam! Weig., Hostr.; Guyana Gallica, Le Pricur! Brasilia! WedDELL.

124. I. commulatum Pr. (Sphaeroc.) p. 34; II. Boryanum Raddi Fil. Brasil. p. 66 tab. 79 fig. 4. (non Willd.)

Hab. Brasilia, Raddi, Mart.!

125. II. Schiedeanum Pr. (Sphaeroc.) p. 34 el 60; H. ciliatum var nudipes $\mathrm{KzE}$ in Linn. XVIII p. 351.

Hab. Mexico, Schiede! Leib., Liebm. alii.

126. II. Boryanum Willd. Sp. pl. V p. 518. Hab. Ins. Bourbon, Bony! Bowin! Mauritins, Sieber! Madagascar, BoIvin!

Species ignolae:

II. trichophyllum HBK. nov. gen. I p. 22. Cumana.

II. diversilobum Pr. Hymen. p. 34 et 59 . Antillae.

11. microcarpum Desv. Ann. Soc. Linn. Paris. VI p. 333. Hispaniola.

II.Sieberi Pr. Hymen. p. 3\% et 58. Martinique. 
127. II. elasticum Bony in Willd. Sp. pl. V p. 520 .

Hab. Ins. Bourbon! Bory, Borvin; Mauritius! Sieber, Borvin; Madagascar! Bernier.

128. H. flavo-aureum Bory in Bec Voy. Ind. or. Bot. p. 84.

Hab. Ins. Bourbon! BoIvin in Herb. de Franqueville.)

Obs. Infausto casu specimina sterilia modo vidi; tamen species adeo videtur insignis, ut, in determinando erraverim, novo nomine serius describenda sit.

129. H.aeruginosum (Porr.) Desv. Ann. Soc. Linn. Paris. VI p. 332; Trichomanes Porr. Encijcl. meth. VIII p. 76. Hab Ins. Tristan d'Acunha, Bory, Carm. (n. v.)

130. II. subtilissimum Kze. (1837 Anal. p. 49); II. Berteroi Ноок. (1846) sp. fil. I p. 93 tab. 33 C.

Hab. Ins. Juan Fernandez, Bert.; Chiloe, Cuming (n. v.)

131. H. Frankliniae Cos. in Tasm. Journ. 1842 p. $376 ;$ H. aeruginosum $\beta$. Ноок. sp. fil. I p. 94.

Hab. Nova Zeelandia, Menz.! CoLEXSO. 
Obs. Species tres ultimas in unam, nomine $\boldsymbol{I}$. aeruginosi, jungendas esse monuit Cl. J. D. Нooker (Fl. N. Zeeland. teste sturm Enum. p. 39). Recte forsan; liceat vero mihi ex mea methodo seorsim illas enumerare, donec frondium contextu bene cognito, notarum externarum diversitatem distinctioni sufficere docuerit, necne. Cautelam suadet preterea speciei, sin est distributio geographica singularis.

132 H. lanceolatum Hook. et ArN. (in BeEcheY Voy.) Hook. Sp. fil. I p. 94 tab. $34 \mathrm{~B}$.

Hab. Ins. Sandvicenses, Gaudicir.! (in Herb. Fée) Lar et Collie, alii.

133. H. oblusum Hook. et ARN. (in BeEcney Voy.) Ноoк. Sp. fil. I p. 93 tab. 33 D.

Hab. Ins. Sandvicenses, Lay et Collie mae! in Herb. Sond. comm. LiNDLEY).

Species ignota:

II. arbuscula Desv. Ann. Soc. Linn. Paris. VI. p. 33?. Ins. Maturitius. 
Desciscentes :

13\%. II. dilalalum (Fonst.) Sw. Syn. p. 1.17 et 373 ; Trichomanes Fonst. Prodr. p.

85 1. 467; Diploophyllum dilata1 lum v. D. B. in sched.

Hab. (Ins. maris pacifici, Forst.); Nov. Zeelandia, Menz., J. D. Ноок.! F. Mülc.! Mossm alii.

135. II. scabrum Ricis. Fl. Nouv. Zeel. p. 90 tab. 14fig. 1; Sphaerocionium glandulife 1 um Pr. Epimel. p. 22 tab. 12; Diploophyllum? scabrum v. D. B. in sched. llab. Nov. Zeelandia, D'Urville, Lyall！J. D. Hook.! Sinclair! alii.

136. II. Chiloënse Ноoк. sp. fil. I p. 90 tab. $32 \mathrm{~A}$; Leptocionium dicranotrichum $\mathrm{Pr}$. Hymen. p. 26 tab. XI D. (teste Sturu Enum. p. 40.)

Hab. Chili, Gay! Leche; ! alii (Chileë, Comixg.

137. I. exsertum Wall. Cat. n. 171; Hook. sp. fil. I. p. 109 tab. 38 A.

Hab. Nepal! WaLl.; mont. Nilagi . rici! Perror.; Ceylon? Tuwaites.

138. II. Gardneri n. sp. Fronde ovata acuminata pinnatifida, laciniis (infimis exceptis) contiguis imbricatisque divaricatis patulisque oblongis inciso-loba- 
tis, lacinulis brevilous subsimplicibus oblongo-linearibus apice attenuato-rotundatisintegris, venis venulisque validis pinnatim ramosis divaricatis, pariter ac rhachis et stipes, pilis elongatis articulatis fuscidulis obsessis, cellulis opacis firmis magnis imo maximis inaequalibus regularibus elongatis acutangulis, parietibus s. rectis s. nodulosis incrassatis, interaneis amorphis olivaceis vel flavo-fuscis, soris in laciniis axillaribus, indusio turgido e basi rotundato-conica suborbiculari mediotenus bilobo, lobis antice grosse crenatis, receptaculo filiformi brevi incluso. Rhizoma intricatum capillaceun paleaceo-strigosum, stipes teres setaceus 1-1, 5 centim. longus, frons 3 centim. longa, 2 centim. circiter lata parum diaphana rigida fusco-olivacea:

Hab. Ins Ceylon, Gardn.! in Herb. Movgeot.

139. H. Zollingeri KzE. in Bot. Ztg. 1848 p. 305; $H$. elasticum Kze. et Zoll. Verz. p. 46. (non Bory)

Hab. Java. ZoLL

140. II. dipleroneuron AL. Br. in Herb; v. D B. in $\mathrm{Pl}$. Jungh. 1. 571 (seorsim p. 27)

Hab. Java! Herb. AL. Br. 
Species ignotae:

11. decurrens JACQ. Coll. III p. 103 tal. 2 fig. 12. Martiniqne.

II. hygrometricum (Poir.) Desv. in Ann. Soc. Par. VI p. 333; Trichomanes PoIn. in Encl. méth. VIII p. 79. Madagascar.

II. Roribundum I. B. K. Nov, gen. I p. 27. Cumana.

II. nigrescens Liغ̇вм. Mex. Bregn. p. 140. Mexico.

II. Telfairianum WalL. Cat. N. 168. Mauritius. 



QL 1.A1 B62 1859

QL 1.A1 B62 1859
Bosch.A.B.van de/Synopsis Hymenophy gen

|| || || |.|.|| || || || || ||

35185001368248 
